\title{
A Statistical Analysis of the Generation of Microseisms
}

\author{
K. Hasselmann \\ Institute of Geophysics and Planetary Physics \\ University of California, San Diego
}

\begin{abstract}
Theories of the origin of microseisms have in the past generally been expressed in terms of the Green functions of the elastic systems considered. An alternative approach based on spectral transfer functions and the local energy-balance equation of the seismic field is proposed. The method enables a rigorous analysis of the statistical aspects of the problem, which could be treated only approximately and under restrictive conditions in terms of the far-field representations used previously. Three suggested origins of microseisms are considered: (1) the action of ocean waves on coasts, originally proposed by Wiechert; (2) atmospheric pressure fluctuations, as suggested by Gherzi, Scholte, and others; and (3) nonlinear interactions between ocean waves as proposed by Longuet-Higgins. In all cases appreciable microseisms are generated only by Fourier components of the random exciting fields that have the same phase velocities as free modes of the elastic system. The effect of pressure fluctuations associated with turbulence in the atmosphere is found to be negligible. The theory for Wiechert's and LonguetHiggins' mechanisms is in good agreement with recent measurements by Haubrich et al.
\end{abstract}

\section{INTRODUCTION}

A number of mechanisms have been proposed to explain the origin of the continuous background noise on seismic records in the range from about 2 to 20 seconds commonly known as microseisms. Wiechert [1904] attributed microseisms to the action of surf on coasts. Gherzi [1924], Scholte [1943], and others assumed that they were generated by pressure fluctuations in the atmosphere. LonguetHiggins [1950] showed that an unattenuated second-order pressure term found by Miche [1944] in standing wave patterns was capable of generating microseisms in deep oceans in which the effect of the attenuated first-order pressure field is normally negligible. The evidence from the large amount of literature on the subject is not always conclusive, although most of the recent work indicates that the majority of observed microseisms can be explained either by Wiechert's or Longuet-Higgins' theory (for summaries, see Gutenberg [1958] and Darbyshire [1962]).

One of the difficulties in determining which mechanism is responsible for observed microseisms by a conclusive quantitative comparison with theory has perhaps been that most of the theoretical analysis has been formulated in terms of the classical response of a given elastic system to a discrete form of excitation, i.e. in terms of the Green function. This method does not lend itself readily to the study of a microseismic field that is generated by a continuous random forcing field. Here the goal of the analysis is not the evaluation of the seismic response to a given discrete excitation but rather the derivation of relations connecting the functions that describe the statistical properties of the seismic field and the generating field. In most applications, these are the power spectra. In principle, 
it is possible to derive from the Green function representation of the seismic field and integral expression relating the covariance functions of the seismic field and the generating field, and then to transform to the power spectra. However, this method is tedious and has not been carried through rigorously, the statistical aspect of the problem having been treated hitherto, if at all, by approximate methods and only for large distances from a finite generating area.

The aim of the present analysis is therefore to introduce an alternative approach to the problem based at the outset on statistical representations and methods. We shall consider first the simplest case of the response of a layered elastic half-space to a random pressure field that is homogeneous and stationary. The field can be assumed to be associated either directly with pressure fluctuations in the atmosphere or with nonlinear gravity-wave interactions. The response in this case is found to be nonstationary owing to the resonant excitation of free modes of the elastic system. The spectra of the modes grow linearly at a rate proportional to the three-dimensional spectral density (with respect to frequency and wave number) of the exciting pressure field at the corresponding resonant points. This is a generalization of Phillips' [1957] result (in the form given by Hasselmann [1962b]) for the rate of growth of a gravity-wave spectrum under the action of a homogeneous, stationary pressure field. Although the idealized case of an infinite, homogeneous, and stationary pressure field is not directly applicable to the real situation, the analysis immediately yields the local rate of energy transfer from the pressure field to the seismic field in the more general case of a random pressure field that is quasi-stationary and quasi-homogeneous. The spatial and temporal variation of the seismic spectra in this case can then be obtained by integrating the differential equation representing the local energy balance of the spectrum under the influence of radiative energy convection and the energy input from the pressure field. The concept of a local energy transfer and energy balance as against the far-field representations obtained by Green function methods enables the determination of the microseismic field at arbitrary positions within or outside the generating area. Refractive effects can also be accounted for in a straightforward manner. Perhaps the main advantage of the method, however, is that it leads to a clearer understanding of the physical nature of the proposed generating mechanisms.

It is found that appreciable microseisms are generated only by the components of the pressure spectrum that have the same phase velocities as free seismic waves. Hence only the high phase-velocity range of the pressure spectrum is important for the problem. It follows from this that Scholte's [1943] evaluation of the amplitude ratios of ocean waves and seismic waves generated by a periodic point source acting on the surface of a fluid layer over an elastic half-space is not relevant for the relative intensities of ocean waves and microseisms generated by random pressure fluctuations. For a random pressure field the ocean waves are generated by relatively slow pressure components in resonance with free gravity waves, whereas the seismic waves are generated by very high phase-velocity components. Scholte's ratio is valid only if the spectral densities in both regions of the pressure spectrum are the same, which is not to be expected. It follows further that the nonlinear pressure term found by Miche [1944] is significant not only because 
it extends to the bottom of a deep layer of fluid but also because it is associated with very high phase velocities capable of exciting seismic waves (in fact, it can be seen that the former property is a direct consequence of the latter). In a similar manner, nonlinear interactions in the atmosphere also result in pressure components with very high horizontal phase velocities. An estimate of the microseisms generated by a turbulent atmospheric boundary layer, however, indicates that atmospheric pressure fluctuations are generally negligible.

For the case of microseisms approaching a beach the analysis has to be modified slightly, since the generating field can no longer be assumed to be approximately homogeneous. However, the microseisms are again found to be generated primarily by pressure components that have the same phase velocity as free seismic waves. Pressure components with these phase velocities are produced by modulation of the low phase-velocity bottom-pressure field as gravity waves pass through shallow water of variable depth. Whereas the effectiveness of the nonlinear interactions increases with frequency, the generation of microseisms by waves approaching a beach is found to decrease rapidly with frequency. Both theories are found to be in satisfactory agreement with recent measurements by Haubrich et al. [1963].

\section{THE GENERATION OF SEISMIC WAVES BY RANDOM PRESSURE FLUCTUATIONS}

Homogeneous, stationary pressure field. Consider the response of a system consisting of a finite number of homogeneous elastic layers over an elastic halfspace under the influence of a random pressure field $p$ acting on the free surface of the system. The case in which the uppermost layer is a fluid is included. We assume that the pressure field is homogeneous and stationary, so that it can be represented as a Fourier-Stieltjes integral

$$
p(\mathbf{x}, t)=\iiint d P(\mathbf{k}, \omega) \exp [i(\mathbf{k} \cdot \mathbf{x}+\omega t)] d \mathbf{k} d \omega
$$

where $\mathbf{x}=\left(x_{1}, x_{2}\right)$ is the horizontal coordinate and $\mathbf{k}$ the corresponding wave number. The power spectrum of $p$ is then

$$
F_{p}(\mathbf{k}, \omega)=\left\langle|d P(\mathbf{k}, \omega)|^{2}\right\rangle /(d \mathbf{k} d \omega)
$$

where cornered brackets denote ensemble means. The compressional and shear waves generated by the pressure component $d P(\mathbf{k}, \omega) \exp [i(\mathbf{k} \cdot \mathbf{x}+\omega t)]$ in each layer $\nu$ can be represented by displacement potentials $d \Phi_{v} \exp [i(\mathbf{k} \cdot \mathbf{x}+\omega t)]$ and $d \Psi, \exp [i(\mathbf{k} \cdot \mathbf{x}+\omega t)]$, where

$$
\begin{aligned}
& d \Phi_{\nu}=d B_{\nu} e^{i k_{\nu^{\prime}} x_{\mathrm{v}}}+d C_{\nu} e^{-\imath k_{\nu^{\prime} x_{\mathrm{s}}}}, \quad k_{\nu}=\sqrt{\frac{\omega^{2}}{\alpha_{\nu}{ }^{2}}-k^{2}} \\
& d \Psi_{\nu}=d D_{\nu} e^{i k_{\nu}{ }^{\prime \prime} x_{\nu}}+d E_{\nu} e^{-i k_{\nu}{ }^{\prime \prime x_{3}}}, \quad k_{\nu}{ }^{\prime}=\sqrt{\frac{\omega^{2}}{\beta_{\nu}{ }^{2}}-k^{2}}
\end{aligned}
$$

and $\alpha_{v}, \beta_{\nu}$ are the compressional- and shear-wave velocities, respectively, in the $\nu$ th layer. The displacements are

$$
d \mathbf{s}_{\nu}=\nabla\left(d \Phi_{\nu} \exp [i(\mathbf{k} \cdot \mathbf{x}+\omega t)]\right)+\nabla \times \frac{\mathbf{k}}{k} \times \mathbf{n}\left(d \Psi_{\nu} \exp [i(\mathbf{k} \cdot \mathbf{x}+\omega t)]\right)
$$


where $\mathbf{n}$ is the unit vector in the direction of the vertical coordinate $x_{3}$, which is measured upward from the free surface of the system. Let us denumerate all amplitudes $d B,-d E$, and denote them simply by the vector $\left(d A_{j}\right)$. The amplitudes are determined by the boundary conditions at the interfaces and at infinity, which yield a linear system of equations (see, e.g., Haskell [1953] and Ewing, Jardetzky, and Press [1957])

$$
b_{i j} d A_{i}=d_{i}\left(d P / \rho_{1} \omega^{2}\right)
$$

Since the external pressure enters only in a single boundary condition at the free surface, only one component of the vector $\left(d_{i}\right)$, say the first, is nonzero. We set $d_{1}=1$. The inclusion of the factor $\rho_{1}^{-1} \omega^{-2}$ (where $\rho_{1}$ is the density of the uppermost layer) in the right-hand side of (1.4) then yields a matrix $b_{i j}$ that is nondimensional and depends only on the phase velocity $\omega / k$ of the pressure component.

The solution of (1.4) is

$$
d A_{i}=\left(B_{1 i} / D\right)\left(d P / \rho_{1} \omega^{2}\right)
$$

where $D=\left|\left(b_{i j}\right)\right|$, and $B_{i j}$ is the subdeterminant matrix of $\left(b_{i j}\right)$.

For the eigenfrequencies $\omega_{1}(\mathbf{k}), \omega_{2}(\mathbf{k}), \omega_{3}(\mathbf{k}), \cdots$, for which $D=0$, the amplitudes become infinite. The number of eigenfrequencies at a given wave number depends on the layer model. The first mode approaches the Rayleigh wave of the elastic half-space as $k \rightarrow 0$. If the propagation velocities increase with the layer depth, as they normally do, the eigensolutions represent waves trapped in the upper layers that are totally reflected at the free surface and at an interface lower down and that interfere constructively. The response of the system near the eigenfrequencies can be obtained by expanding the determinant $D$ in a Taylor series in $\omega^{2}$ about the eigenfrequency $\omega_{n}$ (the matrix $\left(b_{i j}\right)$ is even in $\omega$ ):

$$
D=D_{n}{ }^{\prime}\left(\omega^{2}-\omega_{n}{ }^{2}\right)+\cdots
$$

where

$$
D_{n}{ }^{\prime}=\left(\partial D / \partial \omega^{2}\right)\left(k, \omega_{n}\right)
$$

Thus for $\omega^{2} \approx \omega_{n}^{2}$

$$
d A_{i}=\frac{B_{1 i} d P}{\left(\omega^{2}-\omega_{n}^{2}\right) D_{n}^{\prime} \rho_{1} \omega_{n}^{2}}+\cdots
$$

Equation 1.6 corresponds to the response of an undamped linear oscillator of eigenfrequency $\omega_{n}$ to a force of frequency $\omega$. It can be shown generally [Hasselmann, 19626 t that the response of undamped linear systems to stationary random forces is nonstationary, the energy of the eigensolutions increasing linearly at a rate proportional to the spectral density of the excitation at the resonance frequency. In particular, if $y$ is the solution of the equation

$$
\left(d^{2} / d t^{2}\right) y+\omega_{0}^{2} y=r(t)
$$

for an undamped linear oscillator, in which $r(t)$ is a stationary (not necessarily real) random function, then for large $t$ the power spectrum of $y$ is given by

$$
F_{\nu}(\omega)=\frac{\pi t F_{r}(\omega)}{2 \omega_{0}{ }^{2}}\left\{\delta\left(\omega+\omega_{0}\right)+\delta\left(\omega-\omega_{0}\right)\right\}+\text { const }
$$


where $F_{r}(\omega)$ is the power spectrum of $r$. (Since $y(t)$ is nonstationary, it is meaningful to speak of the spectrum of $y$ only if the relative rate of increase of $|y|^{2}$ is small, i.e. $\left(d|y|^{2} / d t\right)\left(\omega_{0}|y|^{2}\right)^{-1} \ll 1$. This condition follows from equation 1.7 for large $t$.)

Because of (1.7) we obtain from (1.6)

$$
\begin{aligned}
& \left\langle\left|d A_{i}(\mathbf{k}, \omega)\right|^{2}\right\rangle \\
& \quad=\sum_{n} \frac{\pi\left(B_{1 i}\right)^{2} t}{2 \rho_{1}{ }^{2}\left(D_{n}{ }^{\prime}\right)^{2} \omega_{n}{ }^{8}}\left\{\delta\left(\omega-\omega_{n}\right)+\delta\left(\omega+\omega_{n}\right)\right\} F_{p}(\mathbf{k}, \omega) d \mathbf{k}+\text { const }
\end{aligned}
$$

Thus for large $t$ the spectrum $F_{A_{i}}(\mathbf{k}, \omega)=\left|d A_{i}(\mathbf{k}, \omega)\right|^{2} /(d \mathbf{k} d \omega)$ is concentrated entirely on the dispersion surfaces $\omega^{2}=\omega_{n}{ }^{2}(\mathbf{k})$ in $\mathbf{k} \omega$ space. Since $F_{A_{i}}$ is the spectrum of a real variable, it satisfies the relation $F_{A_{i}}(\mathbf{k}, \omega)=F_{A_{i}}(-\mathbf{k},-\omega)$ and can hence be uniquely represented by the set of two-dimensional spectrum $F_{A_{i}}{ }^{(n)}(\mathbf{k})$ obtained by projecting the negative branches $\omega \approx 0$ of the dispersion surfaces on to the $\mathbf{k}$ plane:

$$
F_{A_{i}}{ }^{(n)}(\mathbf{k})=2 \int_{-\omega_{n}-\Delta \omega}^{-\omega_{n}+\Delta \omega} F_{A_{i}}(\mathbf{k}, \omega) d \omega
$$

The definition (1.9) ensures that only waves of the $n$th mode traveling in the positive $\mathbf{k}$ direction contribute to the spectrum $F_{A_{i}}{ }^{(n)}(\mathbf{k})$ at $\mathbf{k}$.

In terms of $F_{A_{i}}{ }^{(n)}(\mathbf{k})$, equation 1.8 can be written

$$
\partial F_{\Delta i}{ }^{(n)}(\mathbf{k}) / \partial t=T_{A_{i}}^{(n)} F_{p}\left(\mathbf{k},-\omega_{n}\right)
$$

where the transfer function

$$
T_{a i}{ }^{(n)}(k)=\frac{\pi\left(B_{1}\right)^{2}}{\rho_{1}{ }^{2}\left(D_{n}^{\prime}\right)^{2} \omega_{n}{ }^{8}}
$$

We note that the left-hand side of (1.10) refers to a singular, two-dimensional spectral distribution on the dispersion surface $\omega=-\omega_{n}(\mathbf{k})$, whereas the righthand side involves the three-dimensional spectral density of $p$ on the surface.

Equation 1.10 is analagous to Phillips' [1957] expression for the rate of growth of ocean waves excited by random pressure fluctuations. In his original paper, Phillips' result is formulated in terms of an autocorrelation integral of the pressure fluctuations. It is shown in Hasselmann [1962c] that this can be reduced to an expression of the form (1.10) by introducing the three-dimensional pressure spectrum and separating components of opposite propagation directions. In a sense, (1.10) includes Phillips' result, since in the case in which the uppermost medium is a fluid the surface gravity waves are some of the normal modes of the system, if gravitational effects are included [Eckart, 1960]. Equation 1.7 (of which equation 1.10 is simply a straightforward generalization) also plays a basic role in the theory of the nonlinear energy transfer in a gravity-wave spectrum [Hasselmann, 1962a, $1963 a, b]$ and probably enters in a similar manner in a number of problems involving the random energy transfer to undamped free modes.

The energy equation. In place of the spectra and transfer functions of the displacement potentials we can substitute in (1.10) the corresponding functions $F_{u}{ }^{(n)}$ and $T_{u}{ }^{(n)}$ of any linear field variable $u$, such as the vertical and horizontal displacements $s_{v}$ and $s_{h}$ of the free surface or (if the uppermost medium is a fluid) of the solid at the fluid-solid interface. 
Of particular interest are the spectrum $F_{\circ}{ }^{(n)}(\mathbf{k})$ and transfer function $T_{\circ}{ }^{(n)}$ of the total energy of the $n$th mode per unit horizontal area. Equation 1.10 then becomes the equation for the energy balance of the $n$th mode and can be generalized to the case normally encountered in practice in which the parameters of the wave guide and the mean properties of the pressure field are only approximately constant. Since the spectrum $F_{\theta}$ in this case is a gradually varying function of position and time, the local energy balance is determined not only by the energy input from the external pressure field but also by the divergence of the inhomogeneous convective energy flux associated with the spectrum. We can interpret the spectrum $F{ }^{(n)}(\mathbf{k}, \mathbf{x})$ as the energy distribution in four-dimensional phase space $\xi=$ $\left(x_{1}, x_{2}, k_{1}, k_{2}\right)$ of a large number of statistically independent wave groups whose dimensions are large in comparison to their wavelength. Since each wave group preserves its energy as it propagates independently along a path $\xi=\zeta(t)$, the convective energy flux in phase space is $\dot{\zeta} F$. Thus the general equation for the energy balance of the $n$th mode is

$$
\frac{\partial F_{e}^{(n)}}{\partial t}+\frac{\partial}{\partial \xi_{e}}\left(\xi_{\imath} F_{e}^{(n)}\right)=T_{e}^{(n)} F_{p}\left(\mathbf{k},-\omega_{n}\right)
$$

Now the propagation paths of wave groups satisfy the well-known Hamiltonian equations

$$
\begin{gathered}
\dot{x}_{i}=\partial \Omega^{(n)} / \partial k_{i} \\
\dot{k}_{i}=-\partial \Omega^{(n)} / \partial x_{i}
\end{gathered}
$$

where $\omega_{n}=\Omega^{(n)}(\mathbf{k}, \mathbf{x})$ is the (spatially dependent) dispersion relationship of the $n$th mode. Since the divergence of $\zeta$ vanishes, (1.12) can be written

$$
\frac{\partial F_{e}^{(n)}}{\partial t}+\dot{x}_{i} \frac{\partial F_{e}^{(n)}}{\partial x_{i}}+\dot{k}_{i} \frac{\partial F_{e}{ }^{(n)}}{\partial k_{i}}=T_{e}^{(n)} F_{p}\left(\mathbf{k},-\omega_{n}\right)
$$

The time derivative $\partial F_{e} / \partial t$ will generally be negligible, since the time required for seismic waves to traverse the pressure field is normally small in comparison with the time scale of variations in the mean pressure field. In the absence of external forces, equation 1.13 yields Longuet-Higgins' [1957] result that the spectrum remains constant along the propagation path of a wave group. The equivalence of this result with Liouville's theorem was pointed out by Dorrestein [1960].

The case of no refraction. Equation 1.13 becomes particularly simple if the refractive term $\dot{k}_{\imath}\left(\partial F_{\bullet}{ }^{(n)} / \partial k_{2}\right)$ vanishes. The analogous energy-balance equation for a gravity-wave spectrum for this case has been considered by Lebel and Gelci [1959], Hasselmann [1960], and Groves and Melcer [1961]. Since the group velocity $\mathbf{v}_{n}=\dot{\mathbf{x}}$ remains constant, $(1.13)$ can be readily integrated:

$$
F_{e}{ }^{(n)}(\mathbf{k}, \mathbf{x}, t)=T_{e}^{(n)}(\mathbf{k}) \int_{t_{0}}^{t} F_{p}\left(\mathbf{k}, \mathbf{x}_{0}+\left(t^{\prime}-t_{0}\right) \mathbf{v}_{n}, t^{\prime}\right) d t^{\prime}+F_{e}^{(n)}\left(\mathbf{k}, \mathbf{x}_{0}, t_{0}\right)
$$

where

$$
\mathbf{x}_{0}=\mathbf{x}-\mathbf{v}_{n}\left(t-t_{0}\right)
$$

In (1.14) the functions $F_{\bullet}{ }^{(n)}$ and $T_{\bullet}^{(n)}$ can again be replaced by the correspond- 


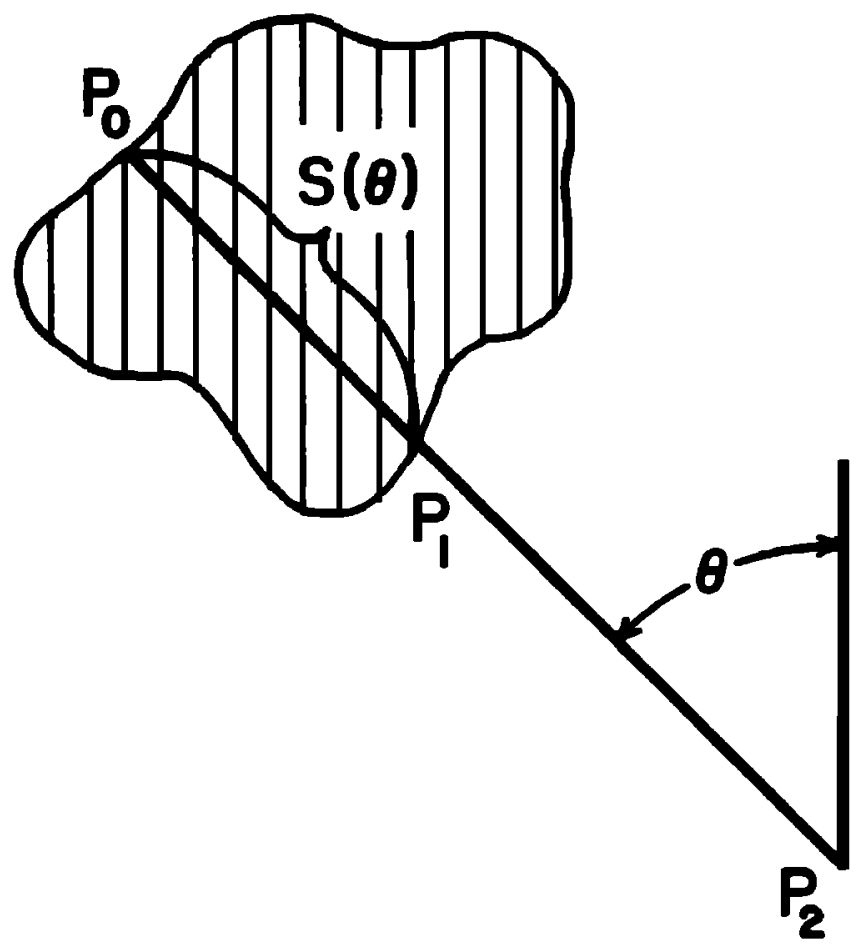

Fig. 1

ing functions for any variable $u$. It is important to note, however, that this is not permissible in the general equation 1.13, since the proportionality factor relating the spectra and transfer functions of different variables depends on $\mathbf{k}$ and therefore cannot be taken before the derivatives if $\mathbf{k}$ varies along the propagation rays.

As an example of the solution 1.14, we consider the simplest case in which $F_{p}\left(\mathbf{k},-\omega_{n}\right)$ is constant within a certain generating area and zero outside (Figure 1$)$. If $P_{0} P_{1} P_{2}$ is the ray corresponding to the wave number $\mathbf{k}$, the spectrum $F_{u}{ }^{(n)}(\mathbf{k})$ grows linearly from its initial value, which we assume to be zero, at $P_{0}$ to a maximal value $\left(s / v_{n}\right) T_{u}{ }^{(n)} F_{p}\left(\mathbf{k},-\omega_{n}\right)$ at the point $P_{1}$ where the ray leaves the generating area (where $s$ is the distance from $P_{0}$ to $P_{1}$ ), and then remains constant along the rest of the ray $P_{1} P_{2}$. In terms of the spectrum $\tilde{F}_{u}{ }^{(n)}(\omega, \theta)=F_{u}{ }^{(n)}(\mathbf{k})\left(k / v_{n}\right)$ with respect to frequency $\omega$ and the propagation direction $\theta$, which is more convenient for experimental purposes,

$$
\tilde{F}_{u, \max }^{(n)}(\omega, \theta)=s(\theta) \tilde{T}_{u}{ }^{(n)}(\omega) \cdot F_{p}\left(\mathbf{k}_{n},-\omega\right)
$$

where

$$
\tilde{T}_{u}^{(n)}(\omega)=\left(k / v_{n}{ }^{2}\right) T_{u}{ }^{(n)}\left(k_{n}\right)
$$

and $\mathbf{k}_{n}$ is the wave number corresponding to $\omega$ and $\theta$ for the $n$th mode.

For large distances from the storm area the spectrum is practically unidirectional and can be adequately described by the one-dimensional frequency spectrum $f_{u}^{(n)}(\omega)=\int_{-\pi}^{+\pi} \tilde{F}_{u}^{(n)}(\omega, \theta) d \theta$. 


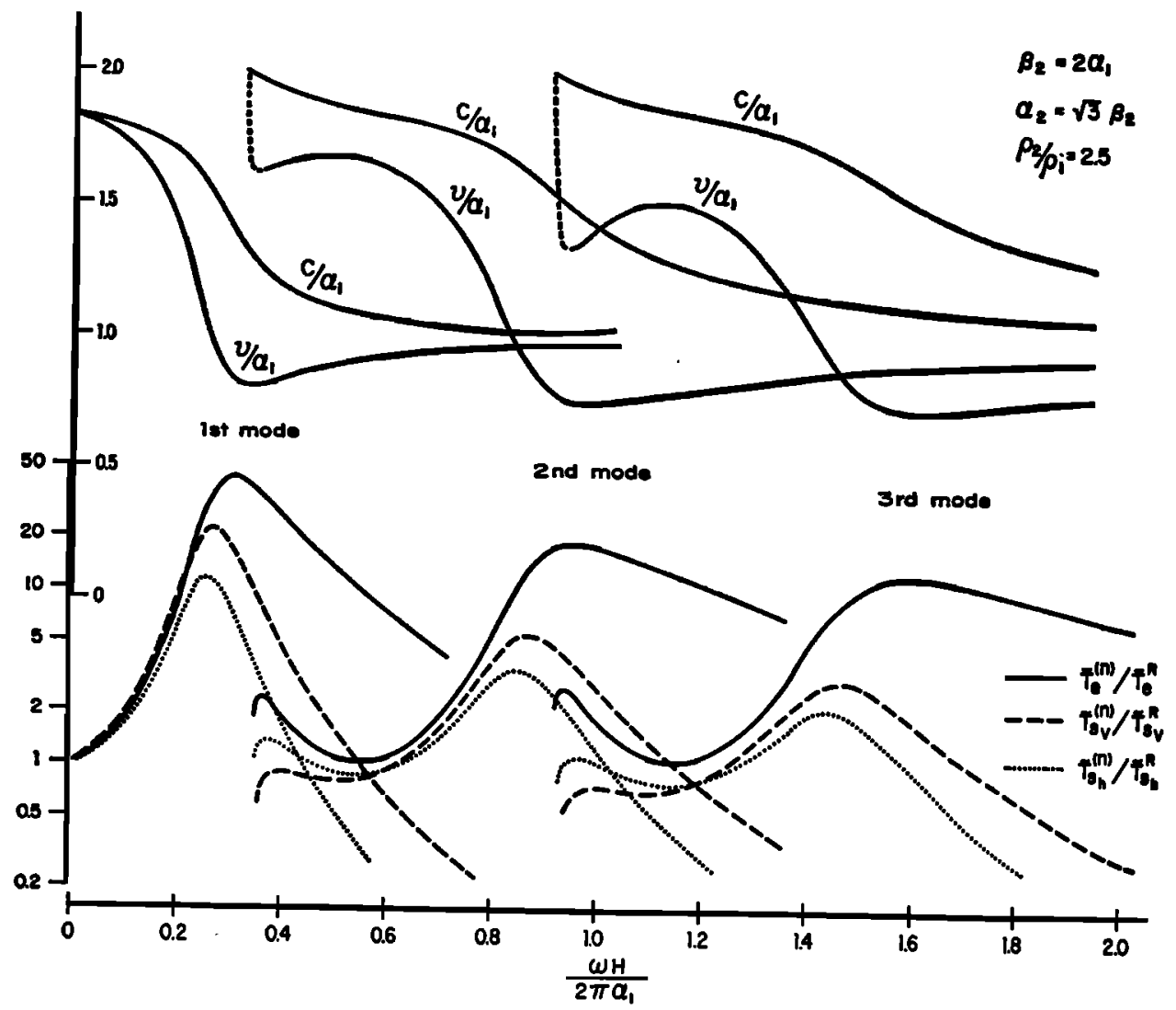

Fig. 2. Top: Dispersion curves for the first three modes of a fluid layer over an elastic halfspace. Bottom: Ratios of the transfer functions $\widetilde{T}^{(n)}$ for the two-layer system to the transfer functions $\tilde{T}^{(R)}$ for the Rayleigh wave of the elastic medium alone. The layer parameters correepond approximately to the media water and granite.

According to (1.15), this is given by

$$
f_{u}^{(n)}(\omega)=(A / R) \tilde{T}_{u}^{(n)}(\omega) F_{p}\left(k_{n},-\omega\right)
$$

where $A$ is the area of, and $R$ the distance from, the generating region.

Equation 1.17 can also be obtained from the asymptotic expansion of the Green function by deriving an integral relation between the covariance functions of the pressure and seismic fields and then transforming to the spectral representations. The relation between $\tilde{T}_{u}{ }^{\left({ }^{n}\right)}$ and the asymptotic response

$$
G_{u}(r, t)=\sum_{n}\left(a_{u}^{(n)} / \sqrt{r}\right) e^{i(k(n) r-\omega t)}+\cdots
$$

of the variable $u$ to a pressure field $p=\delta(\mathbf{x}) e^{-i \omega t}$ acting at the origin (see, e.g., Ewing, Jardetzky, and Press [1957]) is found to be $\tilde{T}_{u}{ }^{\left({ }^{2}\right)}=\left(2 \pi\left|a_{u}{ }^{(n)}\right|\right)^{2}$.

In Figure 2 the transfer functions $\widetilde{T}_{.0}{ }^{(n)}, \widetilde{T}_{e_{b}}{ }^{(n)}$, and $\widetilde{T}_{a}{ }^{(n)}$ for the first three modes are shown together with the dispersion curves for the case of a fluid over an elastic half-space. The elastic constants correspond approximately to water and 
granite. The vertical and horizontal displacements refer to the solid at the fluidsolid interface. The transfer functions are divided by the transfer functions

$$
\begin{aligned}
& \widetilde{T}_{{ }^{2}{ }^{n}}=\omega \cdot \chi_{0_{0}}\left(\alpha_{2} / \beta_{2}\right) \rho_{2}{ }^{-2} \cdot \beta_{2}{ }^{-5} \quad \chi_{0_{0}}(\sqrt{3})=0.460 \\
& \tilde{T}_{\text {oA }}{ }^{R}=\omega \cdot \chi_{\text {sA }}\left(\alpha_{2} / \beta_{2}\right) \rho_{2}{ }^{-2} \cdot \beta_{2}{ }^{-s} \quad \chi_{\text {sA }}(\sqrt{3})=0.214 \\
& \tilde{T}^{{ }^{R}}=\omega^{2} \cdot \chi_{\odot}\left(\alpha_{2} / \beta_{2}\right) \bar{\rho}_{2}^{-1} \cdot \beta_{2}^{-4} \quad \chi_{\odot}(\sqrt{3})=1.36
\end{aligned}
$$

for the Rayleigh mode of the solid half-space in order to bring out the strong amplification due to the water layer. Thus the frequency dependence of the transfer functions $\tilde{T}_{u}{ }^{R}$ has to be taken into account when considering absolute values.

The narrowness of the peaks for the vertical displacement (or of the coefficients $a^{(n)}$ of the Green function) has occasionally been explained as a 'resonance' effect corresponding to the organ-pipe resonance of a column of water over a rigid bottom at wavelength to depth ratios equal to $(n / 2)+(1 / 4), n=1,2, \cdots$. It should be noted, however, that although the sharpness of the peaks is probably related to the relatively high rigidity of the solid medium, the response is due entirely to the resonant excitation of free modes at all frequencies, the maximal response occurring for trapped waves in the fluid layer that have a fairly large angle of incidence.

The amplification is higher for the energy than for the displacements, owing to unequal energy partition in the wave guide. Because of the mismatch in acoustical impedances of the two media, the energy density is considerably higher in the fluid layer than in the solid. Furthermore, the energy density in the fluid is constant, whereas in the solid it decreases exponentially with the distance below the interface. Hence for wavelengths comparable to or greater than the fluid depth the greater part of the wave-guide energy is in the fluid layer. The total energy per unit surface area is consequently higher than for a Rayleigh wave having the same frequency and mean square displacement, the ratio increasing with frequency.

Refraction. In considering the relative effectiveness of microseism generation in the ocean and on land, two bases of comparison are possible. If the microseisms are compared under otherwise identical generating conditions for an elastic halfspace with and without a fluid layer, then the ratios of the displacement spectra are given simply by the ratios of the displacement transfer functions in Figure 2. On the other hand, if the resultant displacement spectra are compared at the same position (say on land), the effects of refraction have to be taken into account and the ratios of the spectra will be determined primarily by the (higher) ratios of the energy transfer functions, since only the energy spectra and not the displacement spectra remain constant during refraction. In other words, as the modes of the fluid-solid system propagate into shallow water, the energy that was initially stored in the fluid layer is gradually transferred to the solid, which leads to a resultant increase in the displacement of the solid surface. The displacement on land is determined finally only by the total energy of the modes. This assumes, of course, that the conversion of energy is gradual and continuous, so that scattering effects are negligible. This is in fact possible only for the first mode, since the energy of higher modes is necessarily scattered as soon as the nondimensional frequency $\omega H / 2 \pi \alpha_{1}$ falls below the low-frequency cutoff (Figure 2). (However, for frequencies below cutoff the higher-order modes can to a first approximation be treated as 
'leaking' modes that decay exponentially [Rosenbaum, 1960; Oliver and Major, 1960; Phinney, 1961; Gilbert and Laster, 1962]. Equation 1.13 can then be generalized to these modes simply by the inclusion of a damping term.) Although the arrival of scattered energy from higher modes may not always be negligible, we shall consider here only the refractive effects for the first mode.

The invariance of the energy spectrum with respect to refraction applies to the spectral density in the wave-number plane. Since the transfer function ratios in Figure 2 refer to the spectral density with respect to frequency and direction, the ratio of the displacement spectra in terms of the latter variables will be proportional not only to the ratio of the energy transfer functions but also to the ratio of the Jakobians $\partial\left(k_{1}, k_{2}\right) / \partial(\omega, \theta)=k / v_{n}=\omega / c_{n} v_{n}$ at the point of observation and in the generating area. Thus, if for the case shown in Figure 1 , for example, we assume that the generating area lies in the ocean, then the spectrum of the displacements, or, more generally, of any variable $u$, on land is

$$
\tilde{F}_{u}{ }^{(1)}\left(\omega, \theta_{R}\right)=s(\theta)\left\{\tilde{T}_{u}{ }^{R}(\omega) \frac{c_{1} v_{1}}{c_{R}{ }^{2}} \frac{\tilde{T}_{\circ}{ }^{(1)}(\omega)}{\tilde{T}_{\bullet}^{R}(\omega)}\right\} F_{\nu}\left(\mathbf{k}_{1},-\omega\right)
$$

where $\theta_{R}$ is the angle of the ray $P_{0} P_{1} P_{2}$ after refraction, $c_{R}$ is the phase velocity of Rayleigh waves, and the index 1 refers to the first mode in the generating area. The ratio $\left(\tilde{T}_{u}{ }^{R} \tilde{T}_{0}{ }^{(1)} / \tilde{T}_{u}{ }^{(1)} \tilde{T}_{\bullet}{ }^{R}\right)\left(c_{1} v_{1} / c_{R}{ }^{2}\right)$ of the expression in braces in (1.18) to the transfer function $\widetilde{T}_{u}{ }^{(1)}$ determining the response before refraction (equation 1.15) is the refraction coefficient (the refraction coefficient is also occasionally defined as the ratio of the amplitudes before and after refraction). If the spectrum is almost unidirectional it is more useful to define the refraction coefficient for the one-dimensional frequency spectrum, which yields an additional factor $d \theta_{R} / d \theta_{\text {. For a }}$ straight shoreline with depth contours parallel to shore, $d \theta_{R} / d \theta=c_{R} \cos \theta / c_{1} \cos \theta_{R}$, where the angles are measured relative to the shore normal. Thus the refraction coefficient is

$$
M_{u}=\frac{\cos \theta v_{1}}{\cos \theta_{R} c_{R}} \tilde{T}_{\stackrel{1}{(1)}}^{\left({ }^{R}\right.} \frac{\tilde{T}_{u}^{R}}{\tilde{T}_{u}^{(1)}}
$$

Since the phase velocity is higher on land than in the ocean, expression 1.19 holds only for $\theta$ smaller than the critical angle $\theta_{\text {orit }}$ for which $\cos \theta_{R}=0$. For $\theta>\theta_{\text {orit }}$ total reflection occurs, and $M_{u}=0$. For $\theta=\theta_{\text {or it }} M_{u}$ becomes infinite. The approximation of a unidirectional spectrum is no longer valid for this angle.

The refraction coefficients $M_{s,}$ and $M_{a b}$ are shown in Figure 3 for normal incidence and the same model as in Figure 2. Also shown is the ratio of the effective transfer function $M_{s,} T_{s_{0}}{ }^{(1)}$ to the transfer function $T_{{ }_{0}}{ }^{(R)}$ for the Rayleigh wave of the elastic half-space. (The ratio for the horizontal component is, of course, the same, since the transfer function $M_{u} T_{u}{ }^{(1)}$ and $T_{u}{ }^{(R)}$ both refer to Rayleigh waves on land.) The amplification due to the transfer of energy from the fluid to the solid is seen to be practically balanced by the higher group velocity on land for frequencies below the frequency of the peaks in Figure 2. Beyond this point, however, the refraction coefficients increase rapidly, so that the peak of the re- 


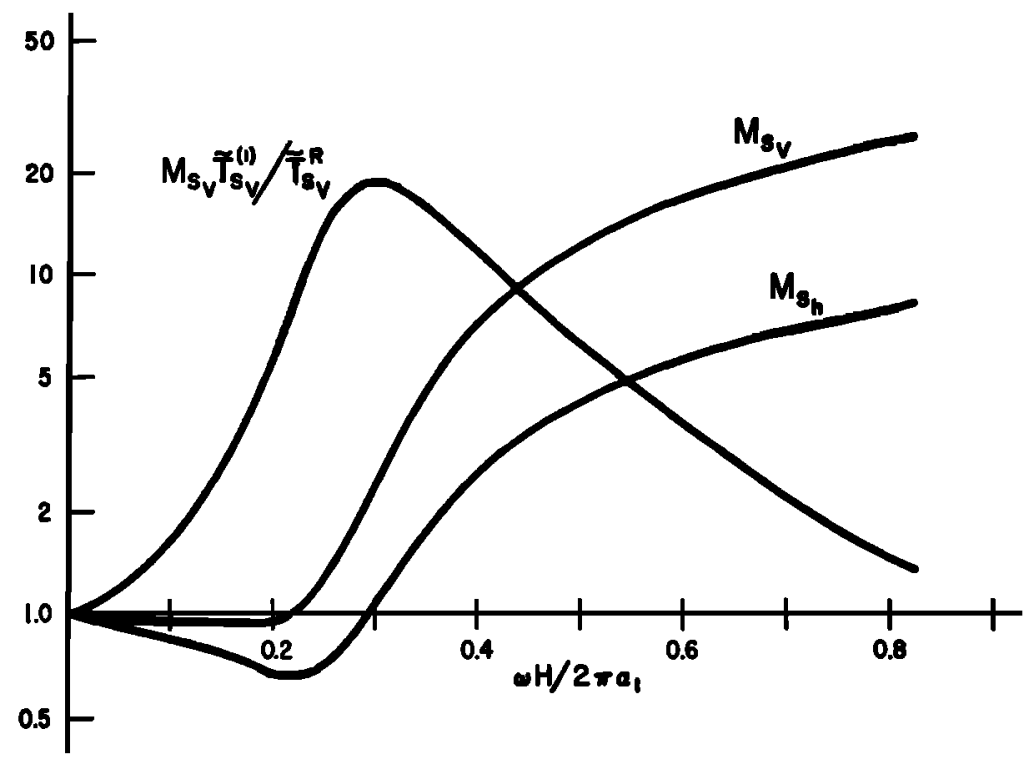

Fig. 3. Refraction coefficients $M_{n,}$ and $M_{n \text {, }}$ for the first mode of a fluidsolid system, and ratio of the net transfer functions $M_{8,} \tilde{T}_{80}$ (1) (including the effect of refraction) for the two-layer system to the transfer function $\widetilde{T}_{a,}(R)$ for the Rayleigh wave of the elastic medium alone. The parameters are as in

Figure 2.

sultant transfer function $M_{0 .} T_{. .}{ }^{(1)}$ is considerably broader than that of $T_{. .}{ }^{(1)}$.

The generating pressure field. We have been concerned so far solely with the response of the elastic system as such and have not inquired into the origin of the exciting pressure field. We found that appreciable microseisms were generated only by pressure components that have the same phase velocities as trapped modes of the wave guide. These velocities are greater than the velocity of sound in both air and water (excluding the gravity-wave modes, if the uppermost medium is a fluid). On the other hand, the velocities of motions in the atmosphere and the ocean are generally well below the sound velocities of the media. Hence we must consider mechanisms by which the energy of these motions can be converted into compressional waves of high phase velocity. The generation of compressional waves by low Mach number turbulence has been investigated by a number of authors [Lighthill, 1952, 1954; Moyal, 1952; Proudman, 1952]. An analogous mechanism by which ocean wave energy is converted into compressional waves has been considered by Longuet-Higgins [1950]. In our analysis we shall use a rather different approach which is one based on the spectral transfer functions rather than on the Green functions of the system.

The mechanism by which energy is converted into high phase-velocity components is basically the same for both turbulent and gravity-wave fields. Quadratic terms in the equations of motion lead to interactions between pairs of Fourier components $\exp \left[i\left(\mathbf{k}_{1} \cdot \mathbf{x}_{1}+\omega_{1} t\right)\right]$ and $\exp \left[i\left(\mathbf{k}_{2} \cdot \mathbf{x}_{2}+\omega_{2} t\right)\right]$, producing a resultant 'sum' component exp $\left\{i\left[\left(\mathbf{k}_{1}+\mathbf{k}_{2}\right) \cdot \mathbf{x}+\left(\omega_{1}+\omega_{2}\right) t\right]\right\}$ (the 'difference' term can be 
regarded as a separate interaction involving the conjugate complex of one of the original components). The phase velocity of the resultant component is then large in comparison to either of the interacting components if the wave numbers $\mathbf{k}_{1}$ and $\mathbf{k}_{2}$, but not the frequencies, are almost equal and opposite. Thus components of high velocity are produced if interactions occur between Fourier components that have almost the same wavelengths and propagation directions but different phase velocities. For ocean waves, where the frequencies and wave numbers are connected by a dispersion relation, this condition can be satisfied only by waves of almost the same frequency traveling in opposite directions. In atmospheric turbulence we shall find a continuum of frequencies associated with the interactions at a given wave number.

In a third example we shall consider a different mechanism in which pressure components of high phase velocity are produced by the linear modulation of the bottom pressure field of gravity waves traveling through shallow water of variable depth.

\section{THE GENERATION OF MICROSEISMS BY NONLINEAR GRAVITY-WAVE INTERACTIONS}

It has been shown by Longuet-Higgins [1950] that second-order interactions between two gravity waves of equal frequency and opposite propagation directions produce a compressional pressure wave that propagates vertically downward through the fluid and is thus capable of exciting elastic waves in the medium beneath. For a rigid bottom Longuet-Higgins found further that the gravitywave interactions were equivalent to a periodic uniform pressure field acting on the surface of the fluid. From our analysis in the preceding section it follows that microseisms are produced, in fact, not by interactions between gravity-wave components having exactly equal and opposite wave numbers, for which the horizontal phase velocity of the generated compressional wave is infinite, but rather by interactions between waves with slightly different wave numbers that produce compressional waves of a finite horizontal phase velocity in resonance with a trapped mode of the wave guide. Since this resonance mechanism tends to be obscured and is difficult to evaluate exactly by the Green function method used by Longuet-Higgins ${ }^{2}$, we shall reinvestigate the problem in terms of the concepts introduced in the preceeding section with the aim of determining rigorously the equivalent pressure spectrum in the general case of a random gravity-wave field in a fluid above a layered elastic half-space. The method has the additional advantage that the response can then be evaluated at any position in the generating area (as in the example considered in the last section) and not only at large distances.

First-order gravity-wave field. To the first order we assume that the motion of the fluid is simply a superposition of incompressible gravity waves satisfying

\footnotetext{
1 In the case of a continuous wave spectrum, Longuet-Higgins considered the interactions between equal and opposite wave numbers of a modified 'blurred' spectrum associated with a limited interaction region. He thus indirectly included interactions between slightly different wave components and obtained a finite trapped-mode component at large distances.
} 
the linearized equations of motion and boundary conditions for an ideal fluid of depth $h$ over a rigid bottom

$$
\begin{aligned}
& \nabla^{2} \phi=0 \text { for }-h<x_{3}<0 \quad \text { (equation of continuity) } \\
& \frac{\partial \phi}{\partial x_{3}}=0 \text { at } x_{3}=-h \quad \text { (kinematical boundary condi- } \\
& \partial x_{3}=0 \text { at } x_{3}=-h \\
& \frac{\partial \zeta}{\partial t}-\frac{\partial \phi}{\partial x_{3}}=0 \text { at } x_{3}=0 \\
& \frac{\partial \phi}{\partial t}+g \zeta=0 \quad \text { at } \quad x_{3}=0
\end{aligned}
$$

where in accordance with the usual notation $\phi$ denotes the velocity potential (rather than the displacement potential used in the preceding section), and $\zeta$ is the surface elevation. We assume that the wave field is random and homogeneous and that the wavelengths are small in comparison to the depth. The solution can then be expressed as Fourier-Stieltjes integrals

$$
\begin{gathered}
\phi\left(\mathbf{x}, x_{3}, t\right)=\iint\left[d \Phi_{+}(\mathbf{k}) e^{-i \sigma t}+d \Phi_{-}(\mathbf{k}) e^{+i \sigma t}\right] \exp \left[i(\mathbf{k} \cdot \mathbf{x})+k x_{3}\right] \\
\zeta(\mathbf{x}, t)=\iint\left[d Z_{+}(\mathbf{k}) e^{-i \sigma t}+d Z_{-}(\mathbf{k}) e^{+i \sigma t}\right] e^{i(\mathbf{k} \cdot \mathbf{x})}
\end{gathered}
$$

where

$\sigma=(g k)^{\frac{1}{3}}$,

$$
\begin{aligned}
d Z_{+}(\mathbf{k}) & = \pm(i \sigma / g) d \Phi_{+}(\mathbf{k}) \\
d \phi_{+}(\mathbf{k}) & =\left(d \phi_{-}(-\mathbf{k})\right)^{*} \\
d Z_{+}(\mathbf{k}) & =\left(d Z_{-}(-\mathbf{k})\right)^{*}
\end{aligned}
$$

The spectrum of the surface elevation, defined in the same way as the spectrum $F_{u}{ }^{(n)}$ in the preceding section to include only waves traveling in the positive $\mathbf{k}$ direction, is then

$$
F_{\zeta}(\mathbf{k})=2\left\langle\left|d Z_{+}\right|^{2}\right\rangle / d \mathbf{k}
$$

Second-order compressional-wave field. In the general case of finite- but small-amplitude gravity waves, we assume that the potential can be expanded in a perturbation series $\phi=\phi_{1}+\phi_{2}+\cdots$, where the first-order term is given by (2.5). To determine $\phi_{2}$ we shall then need to allow not only for the nonlinear terms in the equations of motion and the boundary conditions, but also for the effects of compressibility, since we have seen that the phase velocities of second-order Fourier components can become of the same order as the sound velocity. The continuity equation 2.1 then becomes (for details of the following expansions we refer to Longuet-Higgins [1950])

$$
\nabla^{2} \phi-\frac{1}{\alpha_{1}^{2}}\left(\frac{\partial^{2} \phi}{\partial t^{2}}+g \frac{\partial \phi}{\partial x_{3}}\right)=-\frac{1}{2 \alpha_{1}^{2}} \frac{\partial}{\partial t}(\nabla \phi)^{2}+\cdots
$$

where $\alpha_{1}$ is the sound velocity of the fluid. 
The boundary condition 2.2 for a rigid bottom has to be replaced by the three boundary conditions requiring the continuity of the normal components of the stress tensors and displacements at the fluid-solid interface, together with the equations of motion and the boundary conditions at infinity and at the remaining interfaces of the layered elastic half-space. Since we have assumed that the firstorder gravity-wave motion is negligible at the bottom of the fluid, these equations are linear and homogeneous to the second order. We refer to them as (2.9).

The boundary conditions 2.3 and 2.4 at the free surface become

$$
\begin{gathered}
\frac{\partial \zeta}{\partial t}-\frac{\partial \phi}{\partial x_{3}}=-\frac{\partial \phi}{\partial x_{1}} \frac{\partial \zeta}{\partial x_{1}}-\frac{\partial \phi}{\partial x_{2}} \frac{\partial \zeta}{\partial x_{2}}-\zeta \frac{\partial^{2} \phi}{\partial x_{3}{ }^{2}}+\cdots \quad \text { at } x_{3}=0 \\
\frac{\partial \phi}{\partial t}+g \zeta=-\frac{1}{2}(\nabla \phi)^{2}-\zeta \frac{\partial^{2} \phi}{\partial t \partial x_{3}}+\cdots \quad \text { at } x_{3}=0
\end{gathered}
$$

or, after eliminating $\zeta_{1}$,

$$
\frac{\partial^{2} \phi}{\partial t^{2}}+g \frac{\partial \phi}{\partial x_{3}}=-\frac{\partial}{\partial t}(\nabla \phi)^{2}+\cdots \quad \text { at } \quad x_{3}=0
$$

We note that the solutions of the homogeneous set of equations obtained by setting the right-hand sides of (2.8) and (2.10) equal to zero are the eigensolutions of the layered system, and that the response of the system to a pressure field $p$ acting on the surface of the fluid is obtained by setting the right-hand side of (2.8) equal to zero and the right-hand side of $(2.10)$ equal to $-\left(1 / \zeta_{1}\right)(\partial p / \partial t)$.

The second-order term $\phi_{2}$ of the velocity potential is determined from the second-order term of (2.8)-(2.10), i.e. by substituting $\phi^{(2)}$ and $\phi^{(1)}$, respectively, in the left- and right-hand sides of the equations. The solution $\phi_{2}$ can be represented as the sum of two functions, one of which, $\phi_{2, a}$, is the response of the system to the inhomogeneous term in (2.8) and the other, $\phi_{2, b}$, the response to the inhomogeneous term in (2.10). By substituting the Fourier representation (2.5) of $\phi_{1}$ in the righthand side of $(2.8)$, we readily verifiy that $\phi_{2, a}$ is of the order of $\left(c_{s} / \alpha_{1}\right)^{2}$ smaller than the $\phi_{2, b}$, where $c_{s}$ is the phase velocity of the interacting gravity waves. Hence the solution $\phi_{2}$ is practically determined by the term $-(\partial / \partial t)\left(\nabla \phi_{1}\right)_{x_{3}=0}{ }^{2}$ on the right-hand side of (2.10). From the above remark, this is the same as the response of the system to a pressure field

$$
p=\rho_{1}\left(\nabla \phi_{1}\right)_{x_{3}=0}^{2}
$$

acting on the free surface of the fluid.

The equivalent pressure spectrum. Since the response of the system to a random pressure field has been treated in the preceding section, our problem is thus reduced to the determination of the spectrum $F_{p}(\mathbf{k}, \omega)$ of the equivalent pressure field (2.11). Substituting (2.5) in (2.11) we have

$$
\begin{aligned}
& p=\rho_{1} \sum_{. \mathbf{s}^{\prime \prime}} \int \cdots \int d \Phi_{\mathbf{a}^{\prime}}\left(\mathbf{k}^{\prime}\right) d \Phi_{\mathbf{1}} \cdot\left(\mathbf{k}^{\prime \prime}\right)\left[k^{\prime} \cdot k^{\prime \prime}-\mathbf{k}^{\prime} \cdot \mathbf{k}^{\prime \prime}\right] \\
& \cdot \exp \left\{i\left(\mathbf{k}^{\prime}+\mathbf{k}^{\prime \prime}\right) \cdot \mathbf{x}-i\left(s^{\prime} \sigma^{\prime}+s^{\prime \prime} \sigma^{\prime \prime}\right) t\right\}
\end{aligned}
$$

where $s^{\prime}$ and $s^{\prime \prime}$ denote sign indices. Since $p$ is quadratic in $\varphi_{1}$, the spectrum $F_{p}$ will depend generally on fourth-order moments of $\varphi_{1}$, and cannot be reduced to 
simple statistical functions like the spectrum without further statistical assumptions. However, as $\varphi_{1}$ is the solution of the linearized system, we can without inconsistency assume that $\varphi_{1}$ is Gaussian. Observations and considerations of wave-generating mechanisms indicate that this hypothesis is reasonable. In (2.12) the Fourier amplitudes are then statistically independent, and the pressure spectrum can be expressed in terms of the wave-height spectrum $F_{5}$ :

$$
\begin{aligned}
F_{D}(\mathbf{k}, \omega)=\zeta_{1}{ }^{2} g^{4} \int \cdots \int F_{\zeta}\left(\mathbf{k}^{\prime}\right) F_{\zeta}\left(\mathbf{k}^{\prime \prime}\right)\left(\sigma^{\prime} \sigma^{\prime \prime}\right)^{-2} \\
\cdot\left[\left(k^{\prime} k^{\prime \prime}+\mathbf{k}^{\prime} \cdot \mathbf{k}^{\prime \prime}\right)^{2} \delta\left(\mathbf{k}^{\prime}-\mathbf{k}^{\prime \prime}-\mathbf{k}\right) \delta\left(\sigma^{\prime}-\sigma^{\prime \prime}+\omega\right)\right. \\
\left.+\left(k ! k^{\prime \prime}-\mathbf{k}^{\prime} \cdot \mathbf{k}^{\prime \prime}\right)^{2} \delta\left(\mathbf{k}^{\prime}+\mathbf{k}^{\prime \prime}-k\right) \delta\left(\sigma^{\prime}+\sigma^{\prime \prime}-\omega\right)\right] d \mathbf{k}^{\prime} d \mathbf{k}^{\prime \prime}
\end{aligned}
$$

The general expression 2.13 can be simplified considerably in the range of very high phase velocities responsible for microseism generation. It can be shown that the resultant phase velocity of the first expression (the difference interaction) in the square brackets is always smaller than the phase velocity of a gravity wave of the same wavelength. Since this is in turn smaller than the phase velocity of a seismic wave, the first term does not contribute to the pressure spectrum in the range of interest. If the resultant phase velocity $\left(\sigma^{\prime}+\sigma^{\prime \prime}\right)\left|\mathbf{k}^{\prime}+\mathbf{k}^{\prime \prime}\right|$ of the second term (the sum interaction) in the brackets is large, $\mathbf{k}^{\prime}$ must be approximately equal to $-\mathbf{k}^{\prime \prime}$, so that $\sigma^{\prime} \approx \sigma^{\prime \prime}$. With this approximation, (2.13) reduces to

$$
F_{p}(\mathbf{k}, \omega)=\frac{\rho_{1}{ }^{2} \omega^{7}}{64 g^{2}} \int_{-\pi}^{+\pi} F_{5}\left(\mathbf{k}^{\prime}\right) f_{5}\left(-\mathbf{k}^{\prime}\right) d \theta^{\prime}
$$

where $k^{\prime}=\omega^{2} / 4 g$, and $\theta^{\prime}$ is the angle of $\mathbf{k}^{\prime}$.

In terms of the spectral density $f_{\zeta}(\omega, \theta)=F_{\zeta}(\mathbf{k}) k d k / d \omega=2 F_{\zeta}(\mathbf{k})\left(k^{2} / \omega\right)$ in polar coordinates, $(2.14)$ becomes

$$
F_{\nu}(\mathbf{k}, \omega)=\frac{\zeta_{1}^{2} g^{2} \omega}{2} \int_{-\tau}^{+\pi} f_{\gamma}(\omega / 2, \theta) f_{5}(\omega / 2, \pi+\theta) d \theta
$$

Thus the spectrum $F_{p}(\mathbf{k}, \omega)$ is determined by the integral over all directions of the product of the spectral densities for waves of opposite propagation directions and the same frequency $\omega / 2$. The pressure spectrum is white and isotropic with respect to (in other words, independent of) $\mathbf{k}$. This is to be expected, since the wavelengths of the interacting components are small in comparison with the wavelengths of the generated microseisms. The microseisms generated by a given ocean wave field will, however, generally be anisotropic, since the geometry of the generating area enters in the integration of (1.16). The response at large distances from a finite generating area as determined by (1.20) and (2.15) is in general agreement with the approximate expression derived by Longuet-Higgins [1950].

\section{THE GENERATION OF MICROSEISMS BY ATMOSPHERIC TURBULENCE}

By applying the results of the first section, the determination of the energy transfer from atmospheric turbulence to seismic waves can be reduced, as in the case of an interacting gravity-wave field, to the problem of evaluating the pressure spectrum at the free surface of the layered elastic half-space. We assume that 
pressure fluctuations with phase velocities that are sufficiently high to generate seismic waves are produced by nonlinear interactions in the turbulent boundary layer over the surface. In the frequency range of interest (about 0.03 to $0.5 \mathrm{c} / \mathrm{s}$ ) observations by, for example, Panofsky and Deland [1959] indicate that the boundary layer turbulence is determined primarily by mechanical shear stresses rather than thermal buoyancy forces. For pressure fluctuations of high phase velocity the viscous forces are also small. Neglecting these terms then, the pressure field is determined by the equations of motion

$$
\frac{\partial}{\partial t}\left(\rho u_{\alpha}\right)+\frac{\partial}{\partial x_{\beta}}\left(\rho u_{\beta} u_{\alpha}\right)=-\frac{\partial p}{\partial x_{\alpha}}
$$

and the continuity equation

$$
(\partial \zeta / \partial t)+\left(\partial / \partial x_{\alpha}\right)\left(\rho u_{\alpha}\right)=0
$$

where $\rho$ is the density, and $p$ the variation of the pressure about the value for static equilibrium. Greek indices will be used to denote summation over all three components, whereas Latin indices and the vectors $\mathbf{x}, \mathbf{k}$, etc., refer to vectors in the horizontal plane as hitherto. We shall also denote the three-dimensional wave number later by $\hat{\mathbf{k}}$. We assume that the pressure and density fluctuations are related by the equation $d p / d \rho=\alpha_{0}{ }^{2}$, where the sound velocity $\alpha_{0}$ of the atmosphere can be considered constant within the boundary layer. Equations 3.1 and 3.2 then yield

$$
\frac{1}{\alpha_{0}^{2}} \frac{\partial^{2} p}{\partial t^{2}}-\nabla^{2} p=\frac{\partial^{2}}{\partial x_{\alpha} \partial x_{\beta}}\left(\rho u_{\alpha} u_{\beta}\right)
$$

[Lighthill, 1952]. We assume further that the flow velocities are small in comparison to $\alpha_{0}$. The variation of the density in the right-hand side of (3.3) is then negligible. For brevity, we write $q=\rho\left(\partial^{2} / \partial x_{\alpha} \partial x_{\beta}\right)\left(u_{\alpha} u_{\beta}\right)$.

As boundary conditions we assume that the pressure waves generated by the forcing field $q$ are totally reflected at the bottom of the atmosphere and are dissipated without reflection in the upper atmosphere. For glancing incidence it is known that total reflection of compressional waves can occur at high altitudes, but this is improbable for the small angles of incidence associated with compressional waves whose horizontal phase velocities are as high as those of seismic waves. The first condition implies that the lower boundary of the atmosphere is rigid, or, from (3.1),

$$
\partial p / \partial x_{3}=0 \quad \text { at } x_{3}=0
$$

(This is not, of course, in contradiction with our evaluation of the microseismic field as the necessarily nonrigid response of the layered elastic half-space to the pressure field at $x_{3}=0$. The condition 3.4 implies weak coupling between the atmosphere and the elastic system.) The second condition is equivalent to the Sommerfeld radiation condition.

We assume that the random function $q\left(\mathbf{x}, x_{3}, t\right)$ is homogeneous with respect to $\mathrm{x}$, but not $x_{3}$, and stationary. The covariance function

$$
R_{a}=\left\langle q\left(\mathbf{x}, x_{3}^{\prime}, t\right) q\left(\mathbf{x}+\xi, x_{3}, t+\tau\right)\right\rangle
$$


then depends on the four variables $\xi_{1}, \xi_{2}, x_{3}, x_{3}{ }^{\prime}$ and the time lag $\tau$. In analogy to the case of a random function that is homogeneous with respect to all variables, we define the generalized spectrum $S_{a}$ as the Fourier transform of $R_{q}$ :

$$
\begin{aligned}
S_{a}\left(\mathbf{k}, k_{3}, k_{3}{ }^{\prime}, \omega\right)= & \frac{1}{(2 \pi)^{6}} \int \cdots \int R_{q}\left(\xi, x_{3}, x_{3}{ }^{\prime}, \tau\right) \\
& \cdot \exp \left\{-i\left(\mathbf{k} \cdot \xi+k_{3} x_{3}+k_{3}{ }^{\prime} x_{3}{ }^{\prime}+\omega \tau\right)\right\} d \xi d x_{3} d x_{3}{ }^{\prime} d \tau
\end{aligned}
$$

In terms of the Fourier-Stieltjes representation

$$
\begin{gathered}
q\left(\mathbf{x}, x_{3}, t\right)=\int_{-\infty}^{+\infty} \cdots \int d Q\left(\mathbf{k}, k_{3}, \omega\right) \exp \left\{i\left(\mathbf{k} \cdot \xi+k_{3} x_{3}+\omega t\right)\right\} \\
S_{a}=\frac{\left\langle d Q\left(\mathbf{k}, k_{3}, \omega\right) d Q\left(-\mathbf{k}, k_{3}{ }^{\prime},-\omega\right)\right\rangle}{d \mathbf{k} d k_{3} d k_{3}{ }^{\prime} d \omega}
\end{gathered}
$$

Besides the representation 3.6, it will be convenient to use the Fourier-Stieltjes representation

where

$$
q\left(\mathbf{x}, x_{3}, t\right)=\int_{-\infty}^{+\infty} \cdots \int d \widetilde{Q}\left(\mathbf{k}, x_{3}, \omega\right) \exp \{i(\mathbf{k} \cdot \mathbf{x}+\omega t)\}
$$

$$
d Q\left(\mathbf{k}, k_{3}, \omega\right)=\frac{d k_{3}}{2 \pi} \int_{0}^{\infty} d \widetilde{Q}\left(\mathbf{k}, x_{3}, \omega\right) \exp \left\{-i k_{3} x_{3}\right\} d x_{3}
$$

(note that $d Q$ is a fourth-order and $d \widetilde{Q}$ a third-order differential) and, similarly

$$
p\left(\mathbf{x}, x_{3}, t\right)=\int_{-\infty}^{+\infty} \cdots \int d \tilde{P}\left(\mathbf{k}, x_{3}, \omega\right) \exp \{i(\mathbf{k} \cdot \mathbf{x}+\omega t)\}
$$

The pressure spectrum at $x_{3}=0$ is given by

$$
F_{p}(\mathbf{k}, \omega)=\frac{\left\langle|d \tilde{P}(\mathbf{k}, 0, \omega)|^{2}\right\rangle}{d \mathbf{k} d \omega}
$$

Substituting (3.8) and (3.10) in (3.3), the solution to (3.3) that satisfies the appropriate boundary conditions at $x_{3}=0$ and $x_{3}=\infty$ is found to be

$$
\begin{aligned}
d \widetilde{P}\left(\mathbf{k}, x_{3}, \omega\right)= & -\int_{0}^{x_{2}} \frac{\sin \left[k_{3}{ }^{r}\left(x_{3}-x_{3}{ }^{\prime}\right)\right]}{k_{3}{ }^{r}} d \tilde{Q}\left(\mathbf{k}, x_{3}{ }^{\prime}, \omega\right) d x_{3}{ }^{\prime} \\
& -\frac{2 \pi i d Q\left(\mathbf{k}, k_{3}{ }^{r}, \omega\right)}{d k_{3} \cdot k_{3}{ }^{r}} \cos \left(k_{3}{ }^{r} x_{3}\right)
\end{aligned}
$$

where the vertical wave-number component $k_{3}{ }^{r}$ satisfies the 'resonance conditions'

Thus at $x_{3}=0$

$$
k_{3}{ }^{r}= \pm \sqrt{\frac{\omega^{2}}{\alpha_{0}{ }^{2}}-k^{2}} \text { accordingly as } \omega \gtrless 0
$$

$$
d \widetilde{P}(\mathbf{k}, 0, \omega)=\frac{-2 \pi i}{d k_{3} \cdot k_{3}{ }^{r}} d Q\left(\mathbf{k}, k_{3}{ }^{r}, \omega\right)
$$


According to (3.7) and (3.11) the pressure spectrum at $x_{3}=0$ is then

$$
F_{p}(\mathbf{k}, \omega)=\frac{4 \pi^{2}}{k_{3}^{r^{2}}} S_{a}\left(\mathbf{k}, k_{3}{ }^{r},-k_{3}{ }^{r}, \omega\right)
$$

Equation 3.13 can be compared with the response for an exciting field that is stationary and homogeneous with respect to all variables. As in the derivation of (1.10), it can be shown that in this case the response is nonstationary, the spectrum increasing asymptotically as

$$
F_{p}(\mathbf{k}, \omega)=\frac{t \pi \alpha_{0}}{2 k_{3}{ }^{r}\left(k_{3}^{r^{*}}+k^{2}\right)^{1 / 2}}\left\{F_{a}\left(\mathbf{k}, k_{3}{ }^{r}, \omega\right)+F_{a}\left(\mathbf{k},-k_{3}{ }^{r}, \omega\right)\right\}
$$

where $F_{\varangle}\left(\mathbf{k}, k_{3}, \omega\right)$ is the normal spectral density of a homogeneous, stationary field $q$. The growth of $F_{p}$ is due to the resonant excitation of free pressure waves, the growth rate being proportional to the spectral density of the excitation at the corresponding resonant values of the wave number and frequency. The two terms on the right-hand side of (3.14) correspond to the two possible propagation directions of a free compressional wave with given values of $\omega$ and $\mathbf{k}$.

The relation between (3.13) and (3.14) is clarified when we consider the case of a field $q$ that is almost homogeneous and stationary, so that the spectrum $F_{q}$ can still be approximately defined, but is of finite, although large, extent $L$ in the $x_{3}$ direction. It can be shown from (3.5) that for this field

$$
S_{a}\left(\mathbf{k}, k_{3},-k_{3}, \omega\right) \approx \frac{L}{2 \pi} F_{q}\left(\mathbf{k}, k_{3}, \omega\right)
$$

The equivalence of (3.13) and (3.14) then follows when we observe that the time $t$ for waves to traverse the generating area is $L\left(k^{2}+k_{3}{ }^{{ }^{\prime \prime}}\right)^{1 / 2} / \alpha_{0} k_{k}{ }^{r}$ and that reflection at the lower boundary doubles the amplitudes and thus quadruples the spectrum of the pressure waves. Hence (3.13) is the generalization of (3.14) representing the resonant excitation of free pressure waves to the case in which the random field is inhomogeneous in one direction. For $k_{3}{ }^{r}=0$ the pressure spectrum is infinite. In this case the waves propagate horizontally and remain continually in the propagation region, so that no stationary solution exists. This is of no consequence for the present problem, however, since the horizontal phase velocity of these components $\left(=\alpha_{0}\right)$ is too small to excite seismic waves.

There remains the more difficult problem of expressing the generalized spectrum $S_{a}$ in terms of known statistical properties of the flow field. If we divide the turbulent velocity field into a mean field $\left\langle u_{\alpha}\right\rangle=\bar{u}_{\alpha}\left(x_{3}\right)$ and a fluctuating component $u_{\alpha}{ }^{\prime}$, the exciting field $q$ can be written

$$
\begin{aligned}
q & =\langle q\rangle+q^{\prime} \\
& =\langle q\rangle+\rho \frac{\partial^{2}}{\partial x_{\alpha} \partial x_{\beta}}\left\{u_{\alpha}{ }^{\prime} u_{\beta}{ }^{\prime}-\left\langle u_{\alpha}{ }^{\prime} u_{\beta}{ }^{\prime}\right\rangle+\bar{u}_{\alpha} u_{\beta}{ }^{\prime}+u_{\alpha}{ }^{\prime} \bar{u}_{\beta}\right\}
\end{aligned}
$$

Since the term $\langle q\rangle$ does not contribute to the spectrum we have

$$
S_{\alpha}=k_{\alpha} k_{\beta} k_{\gamma}{ }^{\prime} k_{8}{ }^{\prime} S_{\alpha \beta \gamma \delta}\left(\mathbf{k}, k_{3}, k_{3}{ }^{\prime}, \omega\right)
$$

where $k_{\delta}{ }^{\prime}=-k_{\gamma}, k_{\delta}{ }^{\prime}=-k_{\mathrm{z}}$ for the indices 1 and 2 , and $S_{\alpha \beta \gamma \delta}$ is the (generalized) cross spectrum of the components $T_{\alpha \beta}$ and $T_{\gamma \delta}$ of the fluctuating part

$$
T_{\alpha \beta}=\rho\left[u_{\alpha}^{\prime} u_{\beta}^{\prime}-\left\langle u_{\alpha}{ }^{\prime} u_{\beta}^{\prime}\right\rangle+\bar{u}_{\alpha} u_{\beta}^{\prime}+u_{\alpha}^{\prime} \bar{u}_{\beta}\right]
$$


of the Reynolds stress tensor. The cross spectrum is defined as the Fourier transform of the correlation tensor of $T_{\alpha \beta}$,

$$
\begin{aligned}
S_{\alpha \beta \gamma \delta}=\frac{1}{(2 \pi)^{b}} \int_{-\infty}^{+\infty} & \ldots \int\left\langle T_{\alpha \beta}{ }^{(1)} T_{\gamma \delta}{ }^{(2)}\right. \\
& \cdot \exp \left\{-i\left(\mathrm{k} \xi+k_{8} x_{3}+k_{3}{ }^{\prime} x_{3}{ }^{\prime}+\omega \tau\right) d \xi d x_{3} d x_{3}{ }^{\prime} d \tau\right.
\end{aligned}
$$

where

$$
T_{\alpha \beta}^{(1)}=T_{\alpha \beta}\left(\mathbf{x}+\xi, x_{3}, t+\tau\right) \quad T_{\gamma \delta}^{(2)}=T_{\gamma \delta}\left(\mathbf{x}, x_{3}^{\prime}, t\right)
$$

Since the frequencies and wave numbers in (3.13) correspond to free compressional waves, the wave-number components are at the most equal to $\omega / \alpha_{0}$. For frequencies in the range from 0.2 to $3 \mathrm{rad} / \mathrm{sec}$ this corresponds to wavelengths from about 1 to $15 \mathrm{~km}$. These values will generally be large in comparison with the correlation scales of the turbulent motion at the same frequencies, since the turbulent scales are determined by the much smaller characteristic velocities of the fluid motion rather than the sound velocity. Hence the dependence of the exponent in (3.19) on the spatial coordinates can be neglected, and we can substitute in (3.17) the value of $S_{\alpha \beta \gamma \delta}$ at zero wave number.

Since $T_{\alpha \beta}$ depends on both linear and quadratic expressions of the fluctuating velocity components, $S_{\alpha \beta \gamma \delta}$ depends generally both on fourth-order moments and on the second-order spectra of the velocity fluctuations. It follows from the condition of continuity, however, that the cross spectra of the velocity fluctuations vanish at zero wave number. (See Batchelor [1953] for the proof for homogeneous turbulence. The generalization to our case is straightforward.) Hence

$$
F_{p}(\mathbf{k}, \omega)=C_{\alpha \beta \gamma \delta}(\omega) \frac{k_{\alpha} k_{\beta} k_{\gamma} k_{z}}{\left(k_{3}\right)^{2}}
$$

where $k_{z}=k_{3}{ }^{r}$, as given by (3.12), and

$$
\begin{aligned}
C_{\alpha \beta \gamma \delta}(\omega) & =\frac{\rho^{2}}{(2 \pi)^{3}} \\
\cdot \int_{-\infty}^{+\infty} & \cdots \int\left\langle\left(u_{\alpha}^{\prime} u_{\beta}^{\prime}-\left\langle u_{\alpha}^{\prime} u_{\beta}^{\prime}\right\rangle\right)^{(1)}\left(u_{\gamma}^{\prime} u_{\delta}^{\prime}-\left\langle u_{\gamma}^{\prime} u_{\delta}{ }^{\prime}\right\rangle\right)^{(2)}\right\rangle e^{i \omega r} d \xi d x_{3} d x_{3}{ }^{\prime} d \tau
\end{aligned}
$$

the superscripts (1) and (2) referring to the same coordinates as in (3.19).

We note that the small correlation seale of the generating field relative to a seismic wavelength leads only to the coefficient $C_{\alpha \beta \gamma \delta}$ being independent of $\mathbf{k}$, but not, as for an interacting gravity-wave field, the spectrum itself. This is because the generating pressure field equivalent to the gravity-wave interactions was isotropic, whereas the pressure field is the response to an inhomogeneous quadruple field.

The further evaluation of (3.21) meets with the difficulty encountered generally in problems of aerodynamically generated sound: that very little is known about the fourth-order moments of the turbulent velocity field. To overcome this diffculty it is frequently assumed that the fourth-order moments are related to the 
second-order moments in the same way as if the field were Gaussian. Although the quasi-Gaussian (or, more precisely, zero fourth cumulant) hypothesis is known to be inconsistent with the strongly nonlinear equations of motion and therefore rather questionable when applied to problems involving the detailed dynamical balance of the turbulent field, it may be expected to yield results here that are correct at least to an order of magnitude. Assuming then that

$$
\begin{aligned}
\left\langle\left(u_{\alpha}{ }^{\prime} u_{\beta}\right)^{(1)}\left(u_{\gamma}^{\prime} u_{\delta}^{\prime}\right)^{(2)}\right\rangle=\left\langle\left(u_{\alpha}{ }^{\prime} u_{\beta}{ }^{\prime}\right)^{(1)}\right\rangle\left\langle\left(u_{\gamma}^{\prime} u_{\delta}^{\prime}\right)^{(2)}\right\rangle & +\left\langle\left(u_{\alpha}{ }^{(1)} u_{\gamma}{ }^{(2)}\right)\right\rangle\left\langle u_{\beta}{ }^{(1)} u_{\delta}{ }^{(2)}\right\rangle \\
& +\left\langle\left(u_{\alpha}{ }^{(1)}{u_{\delta}}{ }^{(2)}\right)\right\rangle\left\langle{u_{\beta}}{ }^{(1)} u_{\gamma}{ }^{(2)}\right\rangle
\end{aligned}
$$

equation 3.21 becomes

$$
\begin{aligned}
& C_{\alpha \beta \gamma \delta}(\omega)=\frac{\rho^{2}}{(2 \pi)^{3}} \\
& \cdot \int_{-\infty}^{+\infty} \ldots \int\left\{\left\langle u_{\alpha}{ }^{(1)} u_{\gamma}{ }^{(2)}\right\rangle\left\langle u_{\beta}{ }^{(1)} u_{\delta}{ }^{(2)}\right\rangle+\left\langle u_{\alpha}{ }^{(1)} u_{\delta}{ }^{(2)}\right\rangle\left\langle u_{\beta}{ }^{(1)} u_{\gamma}{ }^{(2)}\right\rangle\right\} e^{i \omega r} d \xi d x_{3} d x_{3}^{\prime} d \tau
\end{aligned}
$$

or, in terms of the generalized cross spectra

$$
\begin{aligned}
& S_{\alpha \beta}\left(\mathbf{k}, k_{3}, k^{\prime}{ }^{\prime}, \omega\right)=\frac{1}{(2 \pi)^{3}} \\
& \cdot \int \cdots \int\left\langle u_{\alpha}{ }^{(1)} u_{\beta}{ }^{(2)}\right\rangle \exp \left\{-i\left(\mathbf{k} \cdot \xi+k_{3} x_{3}+k_{3}{ }^{\prime} x_{3}{ }^{\prime}+\omega \tau\right)\right\} d \xi d x_{3}{ }^{\prime} d x_{3} d \tau \\
& C_{\alpha \beta \gamma \delta}(\omega)=(2 \pi)^{2} \rho^{2} \int_{-\infty}^{+\infty} \cdots \int\left\{S_{\alpha \gamma}\left(\mathbf{k}, k_{3}, k_{3}{ }^{\prime}, \omega^{\prime}\right) S_{\beta \delta}\left(\mathbf{k}, k_{3}, k_{3}{ }^{\prime}, \omega^{\prime}-\omega\right)\right. \\
& \left.+S_{\alpha \delta}\left(\mathbf{k}, k_{3}, k_{3}{ }^{\prime}, \omega^{\prime}\right) S_{\beta \gamma}\left(\mathbf{k}, k_{3}, k_{3}{ }^{\prime}, \omega^{\prime}-\omega\right)\right\} d \mathbf{k} d k_{3} d k_{3}{ }^{\prime} d \omega^{\prime}
\end{aligned}
$$

The reduction to second-order moments has not in itself immediately resolved our difficulties, since measurements of the generalized spectra $S_{\alpha \beta}$ with respect to both wave number and frequency have not been made. However, a rough indication of the spectral distribution is given by Taylor's hypothesis, which states that to a first approximation the turbulent field can be assumed to be simply convected along with the mean stream velocity without changing its spatial structure. If $U$ is the mean velocity, say in the $x_{1}$ direction, the spectrum $S_{\alpha \beta}$ is then concentrated closely around the hyperplane $\omega+U k_{1}=0$ in $\omega \cdot \mathbf{k} \cdot k_{3} \cdot k_{3}{ }^{\prime}$ space. The interdependence of temporal variations and spatial variations in the mean flow direction implied by this relation has been frequently verified experimentally. If the relation were to hold rigorously, the coefficient $C_{\alpha \beta \gamma \delta}$ would be identically zero for $\omega \neq 0$, since it is clearly not possible to satisfy Taylor's relation by both terms of the products in (3.23) simultaneously. However, Taylor's hypothesis can be expected to apply only approximately in our case, since apart from the change in spatial structure of the turbulent field the mean stream velocity is also not constant within the boundary layer. If we assume that the spectrum has a finite spread $\delta \omega=\chi|\omega|$ about the hyperplane $\omega+U k_{1}=0$, the integrand in (3.23) will be different from zero for $\left|\omega^{\prime}\right|>|\omega| / x$. Since we shall be interested 
only in a minimal value of the effective lower limit $\omega_{0}$ of the integration with respect to $\omega^{\prime}$, we set $\chi=1$, which is equivalent to the relatively safe assumption that the spectrum contains no Fourier components whose phase-velocity components in the wind direction are negative.

For $\omega$ in the range from 0.2 to $3 \mathrm{rad} / \mathrm{sec}$ spectral measurements by a number of authors indicate that the lower integration limit $\omega_{0}^{\prime}$ lies within the inertial subrange for most of the boundary layer. Priestley [1959] concludes from several independent measurements that the lower cutoff frequency $\omega_{c}$ of the inertial subrange for the one-dimensional frequency spectrum at the height $x_{3}$ is given approximately by the relation $\omega_{\mathrm{c}} x_{3} / U \approx 5$. Thus for $\omega=1 \mathrm{rad} / \mathrm{sec}$, say, and $U=10$ meters/sec, the contribution to the integral 3.23 will lie within the Kolmogoroff inertial subrange for turbulence above about 50 meters. Since the boundary layer thickness is of the order of $1 \mathrm{~km}$, it can be expected that most of the contribution to $C_{\alpha \beta \gamma \delta}$ will come from turbulence in the inertial subrange. According to Kolmogoroff [Batchelor, 1953] the turbulence in this range is determined solely by the local dissipation $\epsilon\left(x_{3}\right)$ and is quasi-homogeneous and isotropic relative to a system moving with the local mean stream velocity $U\left(x_{3}\right)$. It is thus possible to estimate $C_{\alpha \beta \gamma \delta}$ by using the simplifications associated with the isotropy of the field and applying dimensional analysis. The zero fourth cumulant hypothesis is then no longer strictly necessary, provided that all interactions still occur within the inertial subrange, since the detailed statistical structure of the turbulent field is not relevant for a dimensional argument. On carrying through the analysis, however, the contribution to the coefficient $C_{\alpha \beta \gamma \delta}$ from the turbulence at a height $x_{3}$ is found to be proportional to $\left(\epsilon\left(x_{3}\right)\right)^{7 / 2}$. Since the greater part of the dissipation in a turbulent boundary layer takes place in the lower part of the constant stress layer close to the boundary (see, e.g., Townsend [1956] for general boundary layer flow and Priestley [1959] for the dissipation profile of an atmospheric boundary layer), it appears that $C_{\alpha \beta \gamma \delta}$ is determined primarily by the turbulent field close to the surface, where, because of the smaller scale of the turbulence, it can no longer be assumed that $\omega_{c}{ }^{\prime}$ lies in the inertial subrange. This suggests an alternative dimensional argument, however, for the turbulent field in the lower part of the boundary layer is determined solely by the shear stress $\tau_{0}$ at the boundary. (More precisely, this is so in the logarithmic region of the velocity profile outside the very thin sublayers in which the viscosity and/or the roughness parameter enter as additional independent parameters.) If $C_{\alpha \beta \gamma^{\delta}}$ depends only on the turbulent field in this part of the boundary layer it can be a function only of $\omega$ and $\tau_{0}$, and is hence of the form

$$
C_{\alpha \beta \gamma \delta}(\omega)=\gamma \rho^{2} u_{*}^{8} \omega^{-5}
$$

where $u_{*}=\sqrt{\tau_{0}} / \rho$ is the frictional velocity and $\gamma$ is a constant that we can expect to be of the order of 1 within, perhaps, 2 orders of magnitude. Equation 3.24 can be expected to be valid as long as the distance $5 U / \omega$ is still within the region of the boundary layer controlled by the wall stress.

No account of the tensor character of $C_{\alpha \beta \gamma \delta}$ has been taken in (3.24), but probably the only term of importance in (3.20) is the one involving the vertical components. Since the phase velocities of seismic waves are several times larger 
than the velocity of sound in air, the angles of incidence of atmospheric compressional waves that have the same horizontal phase velocity as seismic waves are small. Hence the wave-number component $k_{3}{ }^{r}$ in $(3.20)$ is considerably larger than the horizontal components, so that the dominant term in the sum is $C_{3333}(\omega) k_{3}{ }^{\prime \prime} \approx C_{\text {zaz3 }}(\omega)\left(\omega^{2} / \alpha_{0}{ }^{2}\right)$. With this approximation we thus have finally

$$
F_{p}(\mathbf{k}, \omega)=\gamma \rho^{2} \alpha_{0}^{-2} u_{*}^{8} \omega^{-3}
$$

As a numerical example we consider the microseisms generated on land by a storm of area $1000 \times 1000 \mathrm{~km}^{2}$ at a distance of $2000 \mathrm{~km}$ at sea. We assume a wind speed of 30 meters/sec, a drag coefficient $U_{*}{ }^{2} / U^{2}$ of 0.0012 , which can be taken as a plausible extrapolation of Deacon's [1962] values obtained at lower wind velocities, and the same two-layered half-space as in section 1 with a water depth of $5 \mathrm{~km}$. Then for a frequency $\omega=0.57 \mathrm{rad} / \mathrm{sec}$ corresponding to the maximum of the net transfer function ratios in Figure 3, we find from (1.17), (1.19), and (3.25)

$$
f_{.0}{ }^{(1)}(\omega)=\gamma 1.2 \cdot 10^{-9} \mu^{2} \text { sec }
$$

Since the observed spectra of microseisms generated by storms are generally of the order of 1 to $10 \mu^{2} \mathrm{sec}$, we conclude that, despite the indeterminancy of the constant $\gamma$ and the approximations involved in deriving (3.25) from (3.20), the generation of microseisms by atmospheric turbulence is generally negligible.

\section{THE GENERATION OF MICROSEISMS BY OCEAN WAVES IN SHALLOW WATER}

The problem of evaluating the microseisms generated by the bottom pressure field of ocean waves traveling through shallow water can conveniently be divided into two parts. First, we consider the response of a layered elastic half-space to a random surface pressure field that is stationary and homogeneous in one direction, say $x_{1}$, but of limited extent in the direction $x_{2}$. A pressure field of this type is produced if waves of a random homogeneous sea pass through a limited shallow water region in which the depth contours run parallel to the $x_{1}$ axis. We then evaluate the pressure field for a given ocean wave spectrum and bottom topography. The analysis will be carried through explicitly only for the case of waves approaching a beach of constant slope, but other cases, corresponding to waves traveling over submerged bars or shelves, will also be considered briefly.

The response to an inhomogeneous, random, bottom pressure field. A pressure field with the stated statistical properties can be represented as a Fourier-Stieltjes integral

$$
p(\mathbf{x}, t)=\int d P(\mathbf{k}, \omega) e^{i(\mathbf{k} \cdot \mathbf{x}+\omega t)}
$$

where the generalized pressure spectrum is

$$
S_{p}\left(k_{1}, k_{2}, k_{2}{ }^{\prime}, \omega\right)=\frac{\left\langle d P\left(k_{1}, k_{2}, \omega\right) d P\left(-k_{1}, k_{2}{ }^{\prime},-\omega\right)\right\rangle}{d k_{1} d k_{2} d k_{2}{ }^{\prime} d \omega}
$$

If $u(\mathbf{x}, t)$ is any linear variable representing the response of the elastic system 
(e.g., a component of the displacement at the free surface) to the pressure field $p$, we then have

$$
u(\mathbf{x}, t)=\int d U(\mathbf{k}, \omega) \exp \{i[(\mathbf{k} \cdot \mathbf{x})+\omega t]\}
$$

where the Fourier components of $u$ and $p$ are linearly related,

with

$$
d U(\mathbf{k}, \omega)=L_{u}(\mathbf{k}, \omega) d P(\mathbf{k}, \omega)
$$

$$
L_{u}(\mathbf{k}, \omega)=\left(L_{u}(-\mathbf{k},-\omega)\right)^{*}
$$

The factor $L_{u}$ becomes infinite at the eigenfrequencies $\omega_{n}(\mathbf{k})$. The singularity can be expressed in terms of the transfer function $T_{u}$ introduced in section 1 . Comparing (1.6) and (1.11) (in which we can replace the potential function by the variable $u$ ), we find

$$
L_{u}(\mathbf{k}, \omega) \approx \frac{\omega_{n}}{\omega^{2}-\omega_{n}^{2}}\left(\frac{T_{u}}{\pi}\right)^{1 / 2} \cdot e^{i \delta_{*} \omega} \text { for } \omega^{2} \approx \omega_{n}^{2}
$$

where $\delta_{n}$ is an undetermined phase factor.

According to (4.4) the covariance function

$$
\begin{aligned}
& R_{u}\left(\xi_{1}, x_{2}, x_{2}{ }^{\prime}, \tau\right)=\left\langle u\left(x_{1}+\xi_{1}, x_{2}, t+\tau\right) u\left(x_{1}, x_{2}{ }^{\prime}, t\right)\right\rangle \\
& =\int \cdots \int\left\langle d U\left(k_{1}, k_{2}, \omega\right) d U\left(-k_{1}, k_{2}{ }^{\prime},-\omega\right)\right\rangle \exp \left[i\left(k_{1} \xi_{1}+k_{2} x_{2}+k_{2}{ }^{\prime} x_{2}{ }^{\prime}+\omega \tau\right)\right]
\end{aligned}
$$

is then

$$
\begin{aligned}
R_{u}\left(\xi_{1}, x_{2}, x_{2}{ }^{\prime}, \tau\right)=\int & \cdots \int L_{u}\left(k_{1}, k_{2}, \omega\right) L_{u}\left(-k_{1}, k_{2}{ }^{\prime},-\omega\right) S_{p}\left(k_{1}, k_{2}, k_{2}{ }^{\prime}, \omega\right) \\
& \cdot \exp \left[i\left(k_{1} \xi_{1}+k_{2} x_{2}+k_{2}{ }^{\prime} x_{2}{ }^{\prime}+\omega \tau\right)\right] d k_{1} d k_{2} d k_{2}{ }^{\prime} d \omega \tau
\end{aligned}
$$

For large values of $x_{2}$ and $x_{2}{ }^{\prime}$ the rapid oscillation of the exponential term practically eliminates all contributions to the integrals with respect to $k_{2}$ and $k_{2}{ }^{\prime}$ except in the immediate neighborhood of the singularities of the factors $L_{u}$. The contribution from the singularities depends on how the integration paths in the complex $k_{2}$ and $k_{2}{ }^{\prime}$ planes are indented around the singularities. This question can be settled in the usual manner by introducing an artificial damping coefficient that is then allowed to approach zero. We find, allowing for (4.5) and (4.6),

$R_{u}\left(\xi_{1}, x_{2}, x_{2}{ }^{\prime}, \tau\right) \rightarrow$

$$
\pi \sum_{n} \iint \frac{\omega^{2}}{\left(k_{2, n}{ }^{2}{ }^{2} c_{n}{ }^{4}\right.} T^{\left({ }^{n}\right)}\left(k_{1}, k_{2, n}{ }^{r}\right) S_{p}\left(k_{1}, k_{2, n}{ }^{r},-k_{2, n}{ }^{r}, \omega\right)
$$

$\cdot \Delta \exp \left[i\left(k_{1} \xi_{1}+k_{2}{ }^{{ }^{2}}\left(x_{2}-x_{2}{ }^{\prime}\right)+\omega \tau\right)\right] d k_{1} d \omega$

where

$$
\Delta=\left\{\begin{array}{l}
1 \\
0 \\
0
\end{array} \text { for } \frac{\omega^{2}}{c_{n}^{2}}-k_{1}^{2}\left\{\begin{array}{l}
>0 \\
<0
\end{array}\right.\right.
$$

where $c_{n}$ is the phase velocity of the $n$th mode for given $\omega_{1}$ and $k_{1}$, and

$$
k_{2, n}{ }^{r}=-\operatorname{sign}(\omega) \sqrt{\left(\omega^{2} / c_{n}{ }^{2}\right)-k_{1}{ }^{2}}
$$


is the second wave-number component of the mode. It has been assumed in (4.8) that $x_{2}$ and $x_{2}{ }^{\prime}$ are both positive; for negative $x_{2}$ and $x_{2}{ }^{\prime}$ the term $k_{2}{ }^{\prime}\left(x_{2}-x_{2}{ }^{\prime}\right)$ in the exponent changes sign. Since for large $x_{2}$ and $x_{2}{ }^{\prime}$ the covariance function $R_{u}$ depends only on the difference coordinates, $u$ is homogeneous and stationary (to moments of second order) and can hence be represented by a normal power spectrum $F_{u}(\mathbf{k}, \omega)$. On comparing (4.8) with the relation

$\left\langle u\left(x_{1}+\xi_{1}, x_{2}+\xi_{2}, t+\tau\right) u\left(x_{1}, x_{2}, t\right)\right\rangle=\iint F_{u}(\mathbf{k}, \omega) \exp [i(\mathbf{k} \cdot \xi+\omega \tau)] d \mathbf{k} d \omega$

connecting the covariance and spectral functions for a homogeneous, stationary variable $u$ we find

$$
F_{u}(k, \omega)=\sum_{n} \frac{\pi \omega^{2}}{k_{2}^{2} c_{n}{ }^{4}} T_{u}\left(k_{1}, k_{2}, \omega\right) S_{\nu}\left(k_{1}, k_{2},-k_{2}, \omega\right) \delta\left(k_{2}-k_{2, n}\right)
$$

According to (4.9) the spectrum is concentrated entirely on the eigenfrequency surfaces $\omega^{2}=\omega_{n}{ }^{2}(\mathbf{k})$ and can thus be expressed, as in section 1 , in terms of the two-dimensional spectra $F_{u}{ }^{(n)}(\mathbf{k})$ or $f_{u}{ }^{(n)}(\omega, \alpha)$. We find

$$
\begin{gathered}
F_{u}{ }^{(n)}(\mathbf{k})= \begin{cases}\frac{2 \pi \omega_{n} v_{n}}{k_{2} c_{n}{ }^{3}} T_{u}{ }^{(n)}(\mathbf{k}) S_{p}\left(k_{1}, k_{2},-k_{2},-\omega_{n}\right) & \text { for } k_{2}>0 \\
0 & \text { for } k_{2}<0\end{cases} \\
f_{u}{ }^{(n)}(\omega, \theta)= \begin{cases}\frac{2 \pi \omega \cdot v_{n}{ }^{2}}{k_{2} c_{n}{ }^{3}} \tilde{T}_{u}{ }^{(n)}(\omega) S_{p}\left(k_{1}, k_{2},-k_{2},-\omega\right) & \text { for } \quad|\theta|<\pi / 2 \\
0 & \text { for } \pi / 2<|\theta|<\pi\end{cases}
\end{gathered}
$$

where the wave-number components on the right-hand side of (4.11) refer to the $n$th mode at the frequency $\omega$ and the propagation direction $\theta$, measured from the $x_{2}$ axis.

Equations 4.10 and 4.11 are analogous to expression 3.13 derived in the preceding section for the pressure response to an inhomogeneous forcing field in a homogeneous medium.

(However, (4.10) and (4.11) hold only for $k_{2} x_{2} \gg 1$, whereas it was possible to derive (3.13) on the immediate boundary of the generating region. The restriction $k_{2} x_{2} \gg 1$ in the general case of a layered medium is due to branch points occurring in the function $L_{u}$.)

The bottom pressure field for waves approaching a constant-slope beach. We turn now to the evaluation of the generalized pressure spectrum $S_{p}$ for the case in which the pressure field is generated by shallow water gravity waves. The condition for the existence of an appreciable bottom pressure field in a fluid of depth $H\left(\omega^{2} H / g=0(1)\right)$ ensures that the influence of the fluid layer on the propagation properties of seismic waves in the layered elastic half-space beneath is negligible ( $\omega H / \alpha \ll 1$, where $\alpha$ is a characteristic propagation velocity of the layered medium), provided that $\omega \alpha / g \gg 1$. Since this is true for frequencies in the range considered, we can neglect the effect of the water layer in determining the seismic response and consider the bottom pressure field to be acting directly on the free surface of the layered elastic half-space.

We assume that in deep water the ocean wave field is homogeneous and can 
thus be represented by the two-dimensional spectrum $F_{5}(\mathbf{k})$ or $f_{5}(\omega, \theta)$ introduced in section 2. For the present purposes it will be convenient to introduce further the spectrum

$$
\hat{F}_{\zeta}\left(k_{1}, \omega\right)=F_{\zeta}(\mathbf{k})\left(2 k^{2} / \omega k_{2}\right)=f_{\zeta}(\omega, \theta) / k_{2}
$$

with respect to the wave-number coordinate $k_{1}$ and (positive) frequency. For the transformation 4.12 to be unique, we assume that $F_{5}(\mathbf{k})=0$ for $k_{2}<0$, so that for a free wave $k_{2}$ is uniquely determined by $\omega_{1}$ and $k_{1}$. The general case is obtained by superimposing on $F_{\zeta}(\mathbf{k})$ a complementary spectrum $\widetilde{F}_{\zeta}(\mathbf{k})$ which is zero for $k_{2}>0$. For simplicity we set $\widetilde{F}_{\zeta}=0$. The Fourier-Stieltjes representation corresponding to the spectrum $\hat{F}_{5}$ is then

$$
\zeta(\mathbf{x}, t)=\iint d Z\left(k_{1}, \omega\right) \exp \left[i\left(k_{1} x_{1}+k_{2}{ }^{\top} x_{2}+\omega t\right)\right]
$$

where $k_{2}{ }^{*}$ is the second wave-number component of the free gravity wave that has the frequency $\omega$ and wave-number component $k_{1}$ and

$$
\widehat{F}_{5}\left(k_{1}, \omega\right)=\frac{\left\langle\left|d Z\left(k_{1},-\omega\right)\right|^{2}\right\rangle}{d k_{1} d \omega}
$$

We assume that the depth contours of the water layer are parallel to the $x_{1}$ axis. Then the Fourier component $d Z\left(k_{1}, \omega\right) \exp \left[i\left(k_{1} x_{1}+k_{2} x_{2}+\omega t\right)\right]$ gives rise to a bottom pressure field with the same periodicity in the $x_{1}$ direction and in time. The pressure amplitude in (4.1) is thus

$$
d P(\mathbf{k}, \omega)=d Z\left(k_{1}, \omega\right) K(\mathbf{k}, \omega) d k_{2}
$$

where

$$
K(\mathbf{k}, \omega)=\frac{1}{2 \pi} \int_{-\infty}^{+\infty} P_{0}\left(x_{2}\right) e^{-i k_{2} x_{2}} d x_{2}
$$

and $P_{0}\left(x_{2}\right) \exp \left[i\left(k_{1} x_{1}+\omega t\right)\right]$ is the bottom pressure field of a wave train that in deep water has unit amplitude, frequency $\omega$, and wave number $\mathbf{k}=\left(k_{1}, k_{2}{ }^{r}\right)$. Equations 4.2, 4.15, and 4.14 then yield

$$
S_{p}\left(k_{1}, k_{2},-k_{2},-\omega_{n}\right)=\hat{F}_{\zeta}\left(k_{1}, \omega_{n}\right)\left|K\left(k_{1}, k_{2},-\omega_{n}\right)\right|^{2}
$$

Since the wavelengths of microseisms are large in comparison with the wavelengths of gravity waves, the seismic wave-number component $k_{1}$ in (4.17) is small in comparison to the component $k_{2}{ }^{\top} \approx \omega_{n}{ }^{2} / g$ of the gravity wave that has a wavenumber component $k_{1}$ and frequency $\omega_{n}$. Hence $\hat{F}_{5}\left(k_{1}, \omega_{n}\right)$ is to a close approximation the spectral density at normal incidence, and, from (4.12),

$$
S_{p}\left(k_{1}, k_{2},-k_{2}, \omega\right) \approx f_{5}\left(\omega_{n}, \theta=0\right) \frac{g}{\omega_{n}^{2}}\left|K\left(k_{1}, k_{2},-\omega_{n}\right)\right|^{2}
$$

where the angle of incidence $\theta$ is measured as in section 1 from the normal to the depth contours.

To evaluate $K(\mathbf{k},-\omega)$ we need to determine the bottom pressure field $P_{0}$ for a harmonic gravity wave at normal incidence. For an arbitrary depth profile this represents a difficult hydrodynamical problem. If we assume, however, that 
the relative change in depth within a distance of the order of a wavelength is small, it is known that to a good approximation the wave field can be treated locally as though the depth $H$ were constant, the variation of the amplitude with $x_{2}$ being determined then from the condition that the energy flux $=$ (amplitude) ${ }^{2} \times$ group velocity remains constant. Thus for a wave of unit amplitude in deep water we have, for normal incidence,

$$
\zeta(\mathbf{x}, t)=Z\left(x_{2}\right) e^{i S\left(x_{2}\right)+i \omega t}
$$

where

$$
\begin{gathered}
d S / d x_{2}=k_{2} \\
Z\left(x_{2}\right)=\sqrt{v_{0} / v}
\end{gathered}
$$

$v_{0}=g / 2 \omega$ is the group velocity in deep water, and the local wave number $k_{2}$ and group velocity $v$ are determined from the dispersion relationship

$$
\omega=\left(g k_{2} \tanh k_{2} H\right)^{1 / 2}
$$

The amplitude of the bottom pressure is then $\rho g Z / \cosh \left(k_{2} H\right)$ [Lamb, 1932], so that

$$
P_{0}\left(x_{2}\right)=\left[\rho g^{3 / 2} /(2 \omega v)^{1 / 2} \cosh \left(k_{2} H\right)\right] e^{i S\left(x_{2}\right)}
$$

The energy argument determines the amplitude $Z\left(x_{2}\right)$ only within an undetermined phase factor that varies slowly in comparison with the phase function $S\left(x_{2}\right)$. Since the wave-number component $k_{2}$ in (4.18) is associated with a free seismic wave, the phase factor in the integrand of (4.16) also varies slowly in comparison to $S\left(x_{2}\right)$ and can consequently be neglected within the approximation of the analysis. Transforming to $S$ as integration variable, we thus have

$$
K\left(k_{1}{ }^{*}, k_{2}^{*},-\omega_{n}\right)=\frac{\rho g^{3 / 2}}{2 \pi \cdot(2 \omega)^{1 / 2}} \int_{-\infty}^{+\infty} \frac{e^{i s} \cdot d S}{v^{1 / 2} \cdot \cosh \left(k_{2} H\right) k_{2}}
$$

where the superscript $s$ has been introduced on the left-hand side of the equation to denote wave-number components associated with seismic waves.

For the case in which the depth varies linearly with $x_{2}$,

$$
H=-H^{\prime} x_{2} \quad\left(x_{2} \leqq 0, H^{\prime}>0\right)
$$

we find that if we introduce the nondimensional variable $y=k_{2} H$ equation 4.23 can be written in the form

$$
K\left(k_{1}{ }^{*}, k_{2}{ }^{*},-\omega_{n}\right)=\frac{\rho g^{2}}{\omega_{n}^{2}} \eta\left(H^{\prime}\right)
$$

where

$\eta\left(H^{\prime}\right)=\frac{1}{2 \pi} \int_{-\infty}^{0} e^{i S}\left\{\tanh y(\cosh y)^{-1}\left[\tanh y+y\left(1-\tanh ^{2} y\right)\right]^{-1 / 2}\right\} d S$

and the variables $S$ and $y$ are connected by

$$
H^{\prime} \frac{d S}{d y}=\left(1+\frac{y}{\tanh y}-y \tanh y\right)
$$




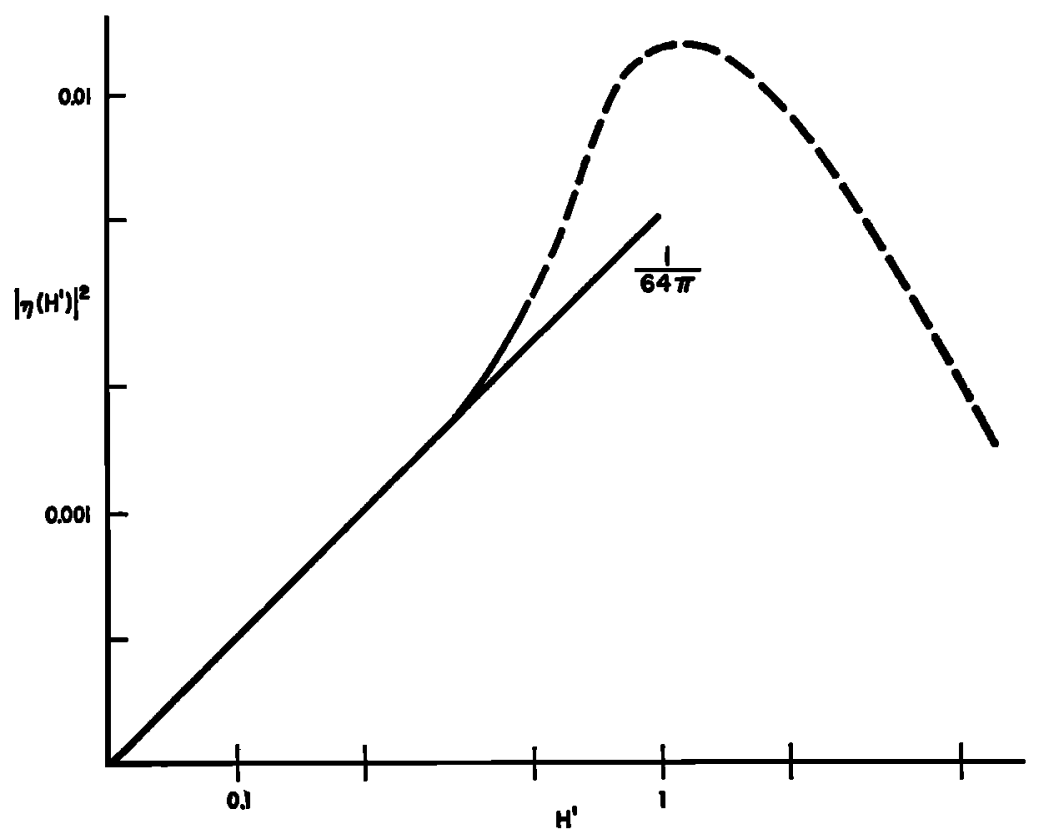

Fig. 4

Although the wave amplitude $Z$ becomes infinite at the shoreline $x_{2}=0$, the integrand in (4.25) remains bounded and indeed approaches zero as $S^{1 / 2} \sim x_{2}{ }^{1 / 4}$. Hence wave breaking need not be invoked as an essential part of the generating mechanism, although we shall find that it may be expected to influence the results numerically.

The computed function $\left|\eta\left(H^{\prime}\right)\right|^{2}$ is shown in Figure 4. Perhaps the most striking feature is the increase of $\left|\eta\left(H^{\prime}\right)\right|^{2}$ with $H^{\prime}$ for small $H^{\prime}$, as it can have been expected intuitively that the energy of the microseisms increases with the area of shallow water in which the gravity waves generate bottom pressure fluctuations. It is apparent from the above analysis, however, that the excitation of seismic waves is due primarily to the modulation of quasi-harmonic pressure waves, which spreads the pressure spectrum over all wave numbers, including, in particular, the range of extremely small wave numbers that is alone effective in microseism generation. Thus the increase of $\left|\eta\left(H^{\prime}\right)\right|^{2}$ with $H^{\prime}$ is due to the stronger modulation associated with more rapid changes in depth.

For small $H^{\prime}$ (which is the only case for which the approximations of our analysis are valid) the function $\left|\eta\left(H^{\prime}\right)\right|^{2}$ can be determined explicitly by expanding (4.25) and (4.26). According to these equations $\eta$ is of the form

$$
\eta\left(H^{\prime}\right)=\int_{-\infty}^{0} e^{i s} \psi\left(S H^{\prime}\right) d S
$$

where for small $x \quad \psi(x)=\left(x^{1 / 2} / 4 \pi\right)\left(1+a_{1} x+a_{2} x^{2}+\cdots\right)$. Hence

$$
\left|\eta\left(H^{\prime}\right)\right|^{2}=\frac{H^{\prime}}{(4 \pi)^{2}}\left|\int_{-\infty}^{0} e^{i S} S^{1 / 2} d S\right|^{2}=\frac{H^{\prime}}{64 \pi}+\cdots
$$




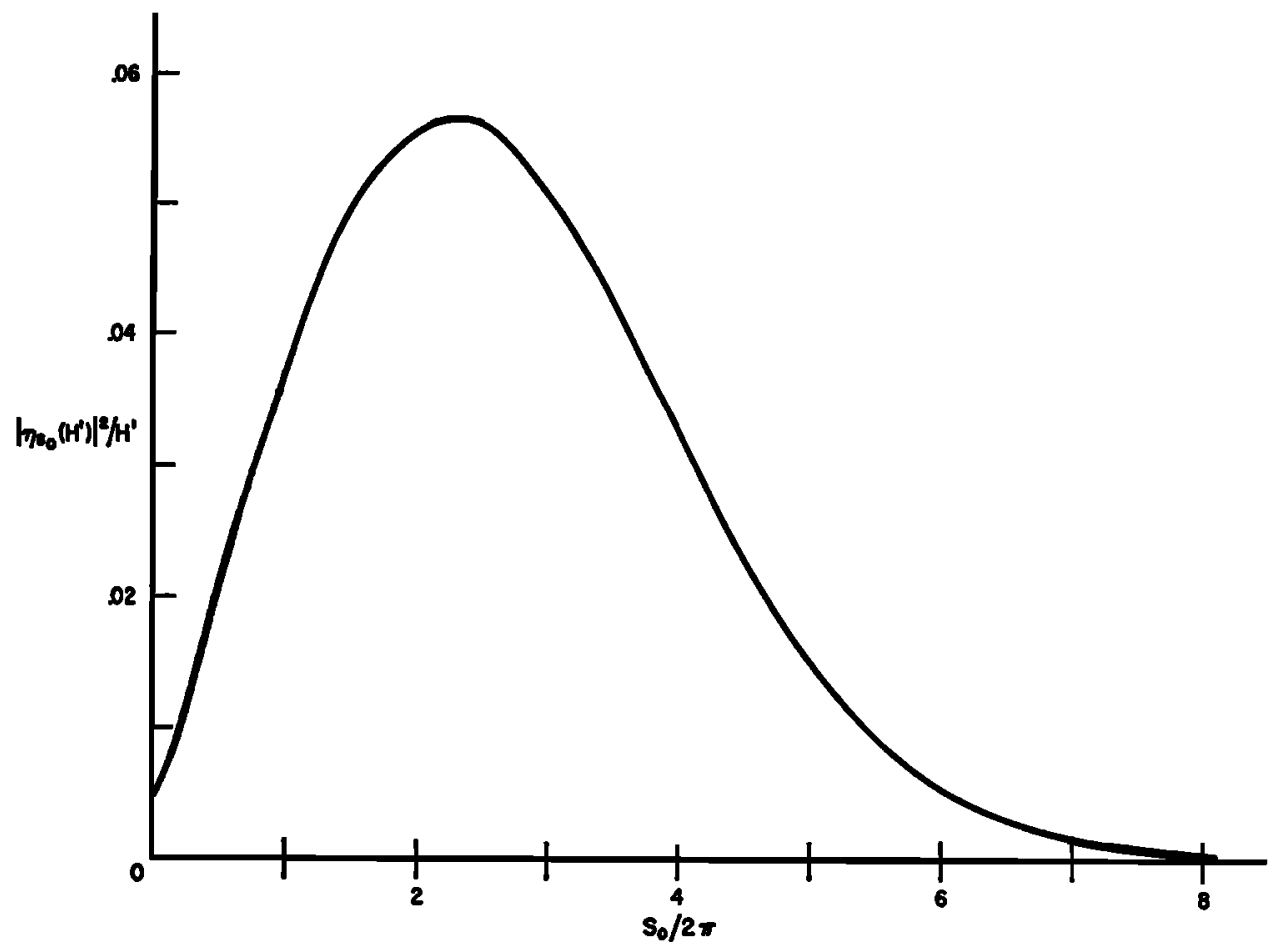

Fig. 5. Amplification due to waves breaking in the idealized case of a sharp cutoff $S_{0} / 2 \pi$ wavelengths from shore.

From (4.11), (4.18), (4.24), and (4.27) we then have finally

$$
f_{u}^{(n)}(\omega, \theta)=\frac{\rho^{2} v_{n}{ }^{2} g^{5} H^{\prime}}{32 \cos \theta c_{n}{ }^{2} \omega^{\theta}} \tilde{T}_{u}^{(n)}(\omega) f_{5}(\omega, 0)
$$

In the case of the Rayleigh mode of an elastic half-space, the displacement transfer functions are proportional to $\omega$ (section 1 ), so that $f_{u}^{\left({ }^{(R)}\right.}(\omega, \theta) \sim \omega^{-5} f_{5}(\omega, 0)$. The strong dependence on $\omega$ favors the generation of low-frequency waves, which may have been a contributing factor for the intense 27-second-period microseisms of gravity-wave origin described by Oliver [1962], and explains generally why surf microseism correlations with a one-to-one frequency ratio are apparently best observed in the low-frequency regions of the spectrum [Oliver and Ewing, 1957].

We have ignored so far the effects of wave breaking. Although these can clearly not be described adequately by a linear theory, an estimate of the influence on the coefficient $K$ can be obtained by considering the extreme case in which the linear theory is assumed to be applicable up to a distance $S_{0} / 2 \pi$ local wavelengths from the shore, at which point the waves are assumed to break and dissipate instantaneously. In place of the function $\left|\eta\left(H^{\prime}\right)\right|^{2}$ given by (4.27), we then have

$$
\left|\eta_{s_{s}}\left(H^{\prime}\right)\right|^{2}=\frac{H^{\prime}}{(4 \pi)^{2}}\left|\int_{-\infty}^{s_{\bullet}} e^{i s} S^{1 / 2} d S\right|^{2}
$$

The function $\left|\eta S_{0}\left(H^{\prime}\right)\right|^{2} / H^{\prime}$ is shown in Figure 5 in dependence on the distance 
(in local wavelengths) from shore of the breaking point. It is seen that for a sharp cutoff two to three wavelengths from shore $\left|\eta_{s_{0}}\left(H^{\prime}\right)\right|^{2}$ is about 10 times as large as the value $\left|\eta\left(H^{\prime}\right)\right|^{2}$ at $S_{0}=0$. This value should be taken as a liberal upper limit, however, as the real situation probably lies, if anything, closer to the case of no breaking at all than the extreme situation considered here.

The bottom pressure field for waves passing over a submerged bar. The higher value of $K$ in the case of a sharp cutoff as compared to the case in which the pressure field extends continuously to the shore line illustrates again the importance of modulation. It may be expected generally that a weakly modulated gravity-wave field without singularities of any form will be considerably less effective in generating microseisms than either of the above cases. As an example that can be assumed characteristic for waves passing over a submerged symmetrical bar we consider the case in which (4.23) is of the form

$$
K\left(k_{1}{ }^{*}, k_{2}{ }^{*},-\omega_{n}\right)=\mu g / \omega_{n}^{2} \int_{-\infty}^{+\infty} e^{i s-\left(S^{*} / 2 \sigma^{2}\right)} d S
$$

where $\mu=(1 / 2 \pi)\left(v_{\infty} / v_{0}\right)^{1 / 2} k_{2, \infty} / k_{2,0}$, the subscripts $\infty$ and 0 referring to values of the group velocity and wave number in deep water and over the center of the bar, respectively, and $\sigma$ is a dimension representing the width of the bottom pressure field in wavelengths. Equation 4.29 yields for this case

$$
|K|^{2}=\left(\mu g / \omega^{2}\right)^{2} 2 \pi \sigma^{2} e^{-\sigma^{2}}
$$

which can be compared with (4.24) and (4.27). For a constant-slope beach the characteristic width in wavelengths of the bottom pressure field is of the order of $\left(H^{\prime}\right)^{-1}$. Hence for bottom pressure fields several times wider than a gravity wavelength, we conclude that, on account of the exponential term in (4.30), the conversion of energy to seismic waves is considerably more effective for waves approaching a beach than for waves passing over a submerged bar. The same conclusion is found for waves passing over a smooth shelf rather than a bar. Since the average slope of the edges of continental shelves is of the order of $4^{\circ}$ to $5^{\circ}$ [Shepard, 1948], it seems improbable that these could be the source of microseisms, as has occasionally been suggested.

\section{COMPARISON WITH MEASUREMENTS}

It has long been observed that ocean waves and microseisms are closely correlated, and numerous measurements have confirmed either a 2 to 1 or 1 to 1 frequency relationship between the spectra (see, e.g., Gutenberg [1958] or Darbyshire [1962]). However, until recently few observations were sufficiently complete to permit a quantitative comparison with the theory. The most detailed measurements to date are probably those of Haubrich et al. [1963]. In a series of measurements extending over six days the authors found sharp peaks at the same frequency in both the gravity-wave and microseismic spectra, and also a strong, slightly broader peak at the double frequency in the microseismic spectrum. All peaks showed a progressive shift to higher frequencies consistent with the linear dispersion of ocean waves generated in a far-distant, short-lived storm (Figure 6). The close agreement between the peak frequencies indicates that the microseisms were generated locally, the double-frequency peak presumably being due to 


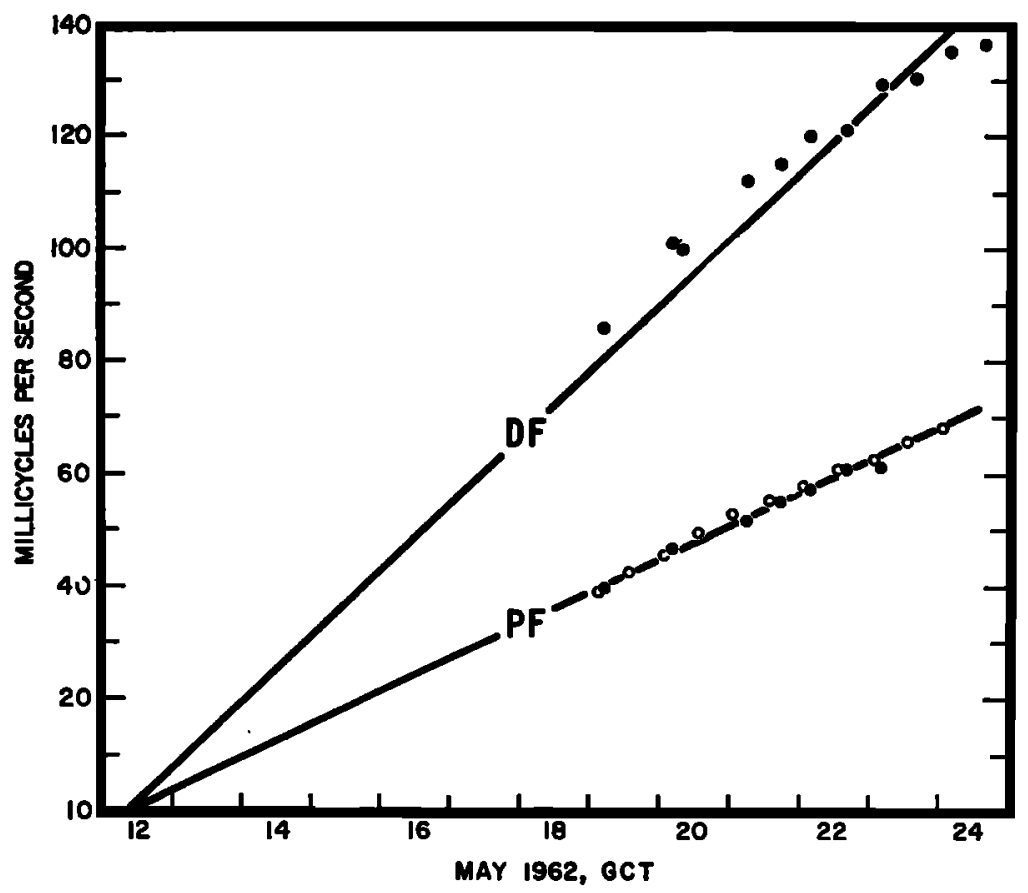

Fig. 6. Dispersive variation in frequency of the spectral peaks of seismic and ocean wave records. The straight lines for the primary frequency $(P F)$ and double frequency $(D F)$ correspond to the theoretical gravity-wave dispersion from a distant source of short duration (from Haubrich et al. [1963]; a few peaks not relevant to the present discussion have not been included).

interactions between the incoming swell and waves reflected from the coast. From the time of origin of the waves (May 12) and the arrival times of the waves, the distance of the storm was found to be $11,500 \mathrm{~km}$. The recording stations for the ocean waves and microseisms were situated close to each other on the south California coast. From previous ocean wave measurements [Munk et al., 1963] the authors suspected a source in the Ross Sea region near $150^{\circ} \mathrm{W}, 60^{\circ} \mathrm{S}$.

The peak spectral densities for successive measurements, and hence increasing frequencies, are shown in Figure 7. The theoretical curves shown for primaryand double-frequency microseisms were calculated from the smooth curve drawn through the ocean wave points. Although measurements of the complete ocean wave and microseismic spectra are presented by Haubrich et al., the theoretical analysis was carried through only for the peaks, as it was doubtful whether the background microseismic spectrum was due entirely to local wave effects. The simple two-layer model of Figures 2 and 3 was taken as the layered elastic halfspace. According to field studies by Shor and Raitt [1958] this should be a reasonable approximation in the area concerned, although a multilayer model would perhaps be more satisfactory. The calculations were based further on the following data and assumptions:

Double frequency. The primary peaks of both ocean wave and seismic spec- 


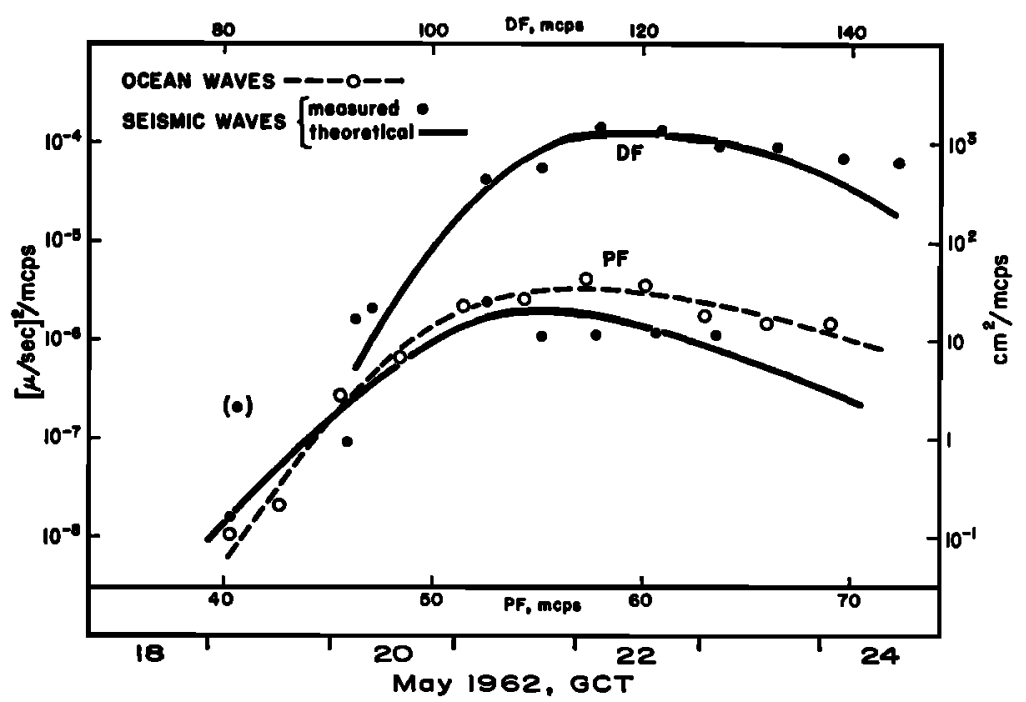

Fig. 7. Experimental and theoretical values of the maximal values of the peaks observed along the dispersion curves of Figure 6 (from Haubrich et al. [1963]).

tra were found to be very narrow; Haubrich et al. give a ratio of $Q=14$ for the peak frequency to peak width at the half-power points for both spectra.

From the dispersion behavior of gravity waves it follows that the duration of the storm which generated the ocean waves was of the order of $Q^{-1} \times$ the travel time of the waves. Similarly, the extent of the generating region in the propagation direction was of the order of $Q^{-1} \times$ the distance traveled by the waves. Assuming the lateral and longitudinal dimensions of the storm to be of the same order, the angular spread of the ocean waves at the recording station is then found to be $11500 / Q R \sin (1150 / R) \approx 8^{\circ}$, where $R=6370 \mathrm{~km}$ is the radius of the earth. For a source in the Ross Sea region the angle of incidence of the beam relative to the shore normal at the observing station is about $30^{\circ} \mathrm{S}$. According to Munk et al. [1963], the energy reflection factor for waves in the frequency range concerned is approximately 0.2 . For a narrow beam imperfectly reflected at an irregular coast, geometrical reflection cannot be expected to hold exactly, and it was consequently assumed that the angular spread of the reflected waves about the reflected mean incident ray was of the order of the rms variation of the shore normal, which was taken as $20^{\circ}$. The spectral distributions with respect to frequency and direction were assumed to be Gaussian in the neighborhood of the peaks. These assumptions determine the local state of the sea at the recording station, so that the spatial distribution of the equivalent pressure spectrum representing the ocean wave interactions can be evaluated from (2.15).

As was shown by Haubrich et al., the effective width of the interaction region is limited in the direction of the incident beam by a relative shift in the peaks of the incident and reflected wave spectra owing to the different travel times of the waves. The width of the interaction region is of the order of $(2 Q)^{-1} \times$ storm distance $\approx 400 \mathrm{~km}$. Since the incident beam is not normal to shore, the dispersive 
shift of the peak also limits the interaction region in the direction parallel to shore to a distance of the same order of magnitude. These dimensions are large in comparison with a seismic wavelength, which in the frequency range concerned is of the order of $25 \mathrm{~km}$. Hence the equivalent pressure field can be regarded as quasihomogeneous, and the microseism spectrum can be determined by integrating the general energy-balance equation 1.13.

The continental borderland off the Californian coast has an average depth of approximately $1 \mathrm{~km}$ for a distance of $300 \mathrm{~km}$ offshore, and then it drops sharply to a depth, including the sediment layer, of $5 \mathrm{~km}$ [Shor and Raitt, 1958]. For a double frequency of $0.1 \mathrm{cps}$ the nondimensional frequency $\omega H / 2 \pi \alpha_{1}$ is then 0.067 on the continental borderland and 0.33 in deep water $\left(\alpha_{1}=1.5 \mathrm{~km} / \mathrm{sec}\right)$. According to Figure 3 the net amplifications due to the water layer are thus 1.3 and 17, respectively. Since the offshore width of the interaction region is $400 \times\left(\cos 30^{\circ}\right)=$ $350 \mathrm{~km}$, most of the interaction region lies on the shelf. However, because of the higher amplification in the deep water, the interactions there are still appreciable. For a Gaussian shaped peak with $Q=14$, we find that the ratio of the contributions from the shelf and the ocean is approximately $1: 2$. This result, however, depends rather strongly on the value of $Q$ and the actual spectral distribution on the flanks of the peak. For higher values of $Q$ the contribution from the ocean rapidly becomes negligible, whereas for lower values of $Q$, as would be associated with waves generated by a nearby storm, the contribution from the ocean is dominant. This explains why the onset of microseismic activity on the east coast of the United States is frequently found to coincide with the passage of a cold front from the continental shelf to deeper water, the peak frequency of the microseisms depending on the depth of the ocean at the position of the front.

Primary frequency. The analysis in the preceding section of microseisms generated by ocean waves approaching a constant-slope beach was based on the model of a perfectly straight shoreline. The model can be assumed suitable for the case of a fairly broad wave beam at small angles of incidence, but is rather unsatisfactory for the present case of a narrow beam at a finite angle of incidence, because the model yielded a rate of microseism generation proportional to the spectral density at zero angle of incidence (equation 4.18), which in the present case is practically zero. Some conversion into seismic energy can nonetheless be expected, owing to the modulation of the bottom pressure field in the parallel-toshore direction on account of irregularities of the coastline. Although the generalization of the analysis required to include this effect is straightforward, it is difficult to determine the relevant properties of the coastline explicitly. It was therefore assumed that to a first order the irregularities of the coastline can be accounted for by an equivalent broadening of the incident wave beam. As in the case of the reflected beam, it was assumed that the beam width was of the same order as the rms variation of the shore normal (which we had taken as $20^{\circ}$ ) and that the angular distribution was Gaussian.

The restriction of the theory to a large distance from shore is also not satisfied in the present case. The seismic recorder was about $16 \mathrm{~km}$ inland, which corresponds approximately to $1 / 3$ of a seismic wavelength at a frequency of $0.05 \mathrm{cps}$. However, for the simple model of an elastic half-space assumed here, the exact response could 
be computed without difficulty, yielding a mean correction factor in the frequency range concerned of $1 / 3$. With a measured mean beach slope of 0.01 at the recording station, good agreement with experiment was then obtained by attributing a correction factor of 3 to wave breaking, consistent with the rough estimate of the previous section.

From the nature of the assumptions required to supplement the experimental data it is clear that the agreement between the experimental and theoretical curves in Figure 7 is significant only to an order of magnitude. The general trend of the curves, however, together with the wide range of spectral densities involved, indicates a satisfactory agreement between theory and experiment.

Acknowledgment. This work has been sponsored in part by the National Science Foundation under grant G-13575.

\section{REFERENCES}

Batchelor, G. K., The Theory of Homogeneous Turbulence, Cambridge University Press, London, 1953.

Darbyshire, J., Microseisms, in The Sea, vol. 1, Physical Oceanography, edited by M. N. Hill, pp. 700-719, Interscience Publishers, New York, 1962.

Deacon, E. L., Aerodynamic roughness of the sea, J. Geophys. Res., 67, 3167-3172, 1962.

Dorrestein, R., Simplified method of determining refraction coefficients for sea waves, J. Geophys. Res., 65, 637-642, 1960.

Eckart, C., Hydrodynamics of Oceans and Atmospheres, Pergamon Press, New York, 1960.

Ewing, W. M., W. S. Jardetzky, and F. Press, Elastic Waves in Layered Media, McGraw-Hill Book Co., New York, 1957.

Gherzi, E., Etude sur les micraséismes, Notes Seismol. Obs. Zi-Ka-Wei, 5, 16 pp., 1924.

Groves, G., and J. Melcer, On the propagation of ocean waves on a sphere, Geofis. Intern., 1, 77-93, 1961.

Gilbert, F., and S. J. Laster, Experimental investigation of PL modes in a single layer, Bull. Seis. Soc. Am., 52, 59-66, 1962.

Gutenberg, B., Microseisms, Advan. Geophys., 5, 53-92, 1958.

Haskell, N. A., Dispersion of surface waves on multilayered media, Bull. Seis. Soc. Am., 43, 17$34,1953$.

Hasselmann, K., Grundgleichungen der Seegangsvoraussage, Schiffstechnik, 7, 191-195, 1960.

Hasselmann, K., On the non-linear energy transfer in a gravity wave spectrum, 1, General theory, J. Fluid Mech., 12, 481-500, 1962a.

Hasselmann, K., Uber Zufallserregte Schwingungssysteme, Z. Angew. Math. Mech., 42, 465$476,1962 b$.

Hasselmann, K., On the non-linear energy transfer in a gravity wave spectrum, 2, Conservation theorems; wave-particle analogy; irreversibility, J. Fluid Mech., 15, 273-281, 1963a; 3 , Evaluation of the energy flux and swell-sea interaction for a Neumann spectrum, $J$. Fluid Mech., 15, 385-398, $1963 b$.

Haubrich, R. A., W. H. Munk, and F. E. Snodgrass, Comparative spectra of microseisms and swell, Bull. Seis. Soc. Am., 58, 27-37, 1963.

Lamb, H., Hydrodynamics, 6th edition, Cambridge University Press, London, 1932.

Lebel, F., and R. Gelci, La prévision numérique de l'état de la mer a l'aide d'un calculateur, J. Mech. Phys. l'Atmosphère, 1, 81-99, 1959.

Lighthill, M. J., On sound generated aerodynamically, 1, Proc. Roy. Soc. London, A, 211, 564$587,1952$.

Lighthill, M. J., On sound generated aerodynamically, 2, Turbulence as a source of sound, Proc. Roy. Soc. London, A, 222, 1-32, 1954. 
Longuet-Higgins, M. S., A theory of the origin of microseisms, Phil. Trans. Roy. Soc. London, $A, 243,1-35,1950$.

Longuet-Higgins, M. S., On the transformation of a continuous spectrum by refraction, Proc. Cambridge Phil. Soc., 53, 226-229, 1957.

Miche, M., Movements ondulatoires de la mer en profondeur cunstante ou décroissante. Ann. Ponts Chaussées, 114, 25-87, 131-164, 270-292, 396-406, 1944.

Moyal, J. E., The spectra of turbulence in a compressible fluid; eddy turbulence and random noise, Proc. Cambridge Phil. Soc., 48, 329-344, 1952.

Munk, W. H., G. Miller, F. E. Snodgrass, and N. F. Barber, Directional studies of ocean waves, Phil. Trans. Roy. Soc. London, A, in press, 1963.

Oliver, J., A world-wide storm of microseisms with periods of about 27 seconds, Bull. Seis. Soc. $A m ., 52,507-517,1962$.

Oliver, J., and M. Ewing, Microseisms in the 11- to 18-second period range, Bull. Seis. Soc. $A m ., 47,111-127,1957$.

Oliver, J., and M. Major, Leaking modes and the PL phase, Bull. Seis. Soc. Am., 50, 165-180, 1960.

Panofsky, H. A., and R. J. Deland, One-dimensional spectra of atmospheric turbulence in the lowest 100 metres, Intern. Symp. Atmospheric Diffusion Pollution, Advan. Geophys., 6, 41-62, 1959.

Phillips, O. M., On the generation of waves by turbulent wind, J. Fluid Mech., 2, 417-445, 1957.

Phinney, R. A., Leaking modes in the crustal wave guide, 1, The oceanic PL wave, J. Geophys. Res., 66, 1445-1469, 1961.

Priestley, C. H. B., Turbulent Transfer in the Lower Atmosphere, University of Chicago Press, Chicago, 1959.

Proudman, I., The generation of noise by isotropic turbulence, Proc. Roy. Soc. London, A, 214, 119-132, 1952.

Rosenbaum, J. H., The long-time response of a layered elastic medium to explosive sound, J. Geophys. Res., 65, 1577-1613, 1960.

Scholte, J. G., Over het verband tussen Zeegloven en microseismen, Verst. Ned. Akad. v. Wetensch., Afd. Natuurkunde, 52, 669-683, 1943.

Shepard, F. P., Submarine Geology, Harper and Brothers, New York, 1948.

Shor, G. G., Jr., and R. W. Raitt, Seismic studies in the southern California continental borderland, Congr. Geol. Intern., Secc. 9, Geofis. Appl., 2, 253-259, 1958.

Townsend, A. A., The Structure of Turb:clent Shear F'lnw, Cambridge University Press, London, 1956.

Wiechert, E., Verhandlungen der Zweiten Internationalen Seismologischen Konferenz. Gerl. Beitr. Geophys., Ergänzungsbd. 2, 41-43, 1904.

(Manuscript received January 15, 1963; revised February 6, 1963.) 